\title{
NON-REDUCTIVE HOMOGENEOUS PSEUDO-RIEMANNIAN MANIFOLDS OF DIMENSION FOUR
}

\author{
M.E. Fels, A.G. Renner \\ Department of Mathematics and Statistics, Utah State University, Logan Utah, 84322
}

October 12, 2004

\begin{abstract}
A method, due to Élie Cartan, is used to give an algebraic classification of the non-reductive homogeneous pseudo-Riemannian manifolds of dimension four. Only one case with Lorentz signature can be Einstein without having constant curvature, and two cases with $(2,2)$ signature are Einstein of which one is Ricci-flat. If a four-dimensional non-reductive homogeneous pseudo-Riemannian manifold is simply connected, then it is shown to be diffeomorphic to $\mathbb{R}^{4}$. All metrics for the simply connected non-reductive Einstein spaces are given explicitly. There are no non-reductive pseudoRiemannian homogeneous spaces of dimension two and none of dimension three with connected isotropy subgroup.
\end{abstract}

\section{INTRODUCTION}

A homogeneous space $G / H$, where $G$ is a Lie group and $H$ a closed Lie subgroup, is reductive [9] if the Lie algebra $\mathbf{g}$ of $G$ may be decomposed into a vector-space direct sum $\mathbf{g}=\mathbf{h} \oplus \mathbf{m}$ where $\mathbf{m}$ is an $\operatorname{Ad}(H)$-invariant complement to $\mathbf{h}$. If $G / H$ is a reductive homogeneous space which admits a pseudo-Riemannian metric with $G$ acting by isometries, the curvature tensor takes on a particularly simple form. For this reason, the geometry of these spaces has been well studied [9] [2], and some classification results are known [5]. On the other hand, little is known about the structure of nonreductive homogeneous pseudo-Riemannian manifolds and the purpose of this paper is classify and investigate the basic geometry and topology of these special manifolds in low dimensions.

While it is easy to construct non-reductive homogeneous spaces, it is quite a bit more difficult to construct examples where $G$ is the isometry group of a pseudo-Riemannian metric on $G / H$. The difficulty is that if $G$ is the isometry group of a Riemannian metric on $G / H$ then $\operatorname{Ad}(H)$ is compact, so $G / H$ is automatically reductive (see section 4 for an algebraic proof). Therefore, to construct examples of non-reductive pseudo-Riemannian homogeneous spaces only metrics with indefinite signature need to be considered. These facts are mentioned in [2], but no non-reductive examples are given. In the article [11] the author studies the ring of invariant differential operators on non-reductive homogeneous spaces but only considers geometric examples which turn out to be reductive.

In the book Leçons sur la géométrie des espaces de Riemann [3], Cartan outlines a method in which questions about the geometry of homogeneous Riemannian manifolds become algebraic

1991 Mathematics Subject Classification. Primary 53C30.

Key words and phrases. Homogeneous pseudo-Riemannian, Einstein space. 
questions about Lie algebras. Cartan used his technique to classify the three-dimensional simply connected Riemannian homogeneous spaces which admit a group of isometries of dimension at least 4. Ishihara [7] used Cartan's method to classify the four-dimensional Riemannian manifolds with transitive isometry groups while Jensen [8] used this technique to determine the simply connected homogeneous Einstein spaces of dimension 4. An alternative approach to the classification of low dimensional homogeneous Riemannian manifolds was given in [1], but this approach utilizes the compactness of the isotropy subgroup and so can't be used here.

Cartan's method works perfectly well for pseudo-Riemannian homogeneous spaces. We use this method in section 5 to first show that there are no two or three-dimensional non-reductive homogeneous pseudo-Riemannian manifolds. We then classify the four-dimensional non-reductive homogeneous pseudo-Riemannian manifolds and show in section 6 that if these four-dimensional homogeneous spaces are simply connected then they are diffeomorphic to $\mathbb{R}^{4}$. As a consequence of the calculations in section 5, we identify the cases which are Einstein and compare them with those in [10]. Finally, in section 7 we construct the corresponding homogeneous Einstein metrics on $\mathbb{R}^{4}$ (the simply connected spaces) for the three cases in which they exists.

\section{The Classification and Einstein metrics}

In this section, we provide a summary of the classifications proved in section 5 and then list the possible Einstein metrics which are found in section 7 when $G / H$ is assumed to be simply connected.

If $\eta$ is a pseudo-Riemannian metric on the manifold $G / H$ and $G$ acts effectively and by isometries, we say the pair $(G / H, \eta)$ is a homogeneous pseudo-Riemannian manifold. We also use the convention that the bilinear form $\eta$ on an $n$-dimensional Lorentz manifold has signature $(n-1,1)$.

Theorem 2.1. Let $(G / H, \eta)$ be a homogeneous Lorentz manifold. If $G / H$ is two-dimensional, then $G / H$ is reductive. If $G / H$ is three-dimensional and $H$ is connected, then $G / H$ is reductive.

Let $\mathbf{h}$ be a Lie subalgebra of the Lie algebra $\mathbf{g}$ and denote this pair by $(\mathbf{g}, \mathbf{h})$.

Definition. The Lie algebra pairs $(\mathbf{g}, \mathbf{h})$ and $\left(\mathbf{g}^{\prime}, \mathbf{h}^{\prime}\right)$ are isomorphic if there exists an isomorphism $\Phi: \mathbf{g} \rightarrow \mathbf{g}^{\prime}$ such that $\Phi(\mathbf{h})=\mathbf{h}^{\prime}$.

For every homogeneous space $G / H$, let $\mathbf{g}$ be the the Lie algebra of $G$ and $\mathbf{h}$ the Lie algebra of $H$, let $(\mathbf{g}, \mathbf{h})$ be the associated Lie algebra pair. In the next theorem, we list all possible non-isomorphic Lie algebra pairs for the non-reductive four-dimensional homogeneous spaces that are classified in section 5. We use the table of Lie algebras in [17] and refer to these algebras by $A_{x, y}$, as given on page 990 .

Theorem 2.2. Let $(G / H, \eta)$ be a four-dimensional homogeneous Lorentz manifold where $H$ is connected. If $G / H$ is not reductive, then the Lie algebra pair $(\mathbf{g}, \mathbf{h})$ is isomorphic to one in the following list.

A1 The Lie algebra $\mathbf{g}$ is the decomposable five-dimensional algebra $\mathbf{s}(2, \mathbb{R}) \oplus \mathbf{s}(2)$, where $\mathbf{s}(2)$ is the two-dimensional solvable algebra. There exists a basis for $\mathbf{g}$ where the non-zero products are

$$
\left[\mathbf{e}_{1}, \mathbf{e}_{2}\right]=2 \mathbf{e}_{2},\left[\mathbf{e}_{1}, \mathbf{e}_{3}\right]=-2 \mathbf{e}_{3},\left[\mathbf{e}_{2}, \mathbf{e}_{3}\right]=\mathbf{e}_{1},\left[\mathbf{e}_{4}, \mathbf{e}_{5}\right]=\mathbf{e}_{4} .
$$

The isotropy is $\mathbf{h}=\operatorname{span}\left\{\mathbf{e}_{3}+\mathbf{e}_{4}\right\}$.

A2 The Lie algebra $\mathbf{g}$ is the one-parameter family of five-dimensional solvable Lie algebras $A_{5,30}$. There exists a basis for $\mathbf{g}$ where the non-zero products are

$$
\begin{array}{lll}
{\left[\mathbf{e}_{1}, \mathbf{e}_{5}\right]=(\alpha+1) \mathbf{e}_{1},} & {\left[\mathbf{e}_{2}, \mathbf{e}_{4}\right]=\mathbf{e}_{1},} & {\left[\mathbf{e}_{2}, \mathbf{e}_{5}\right]=\alpha \mathbf{e}_{2},} \\
{\left[\mathbf{e}_{3}, \mathbf{e}_{4}\right]=\mathbf{e}_{2},} & {\left[\mathbf{e}_{3}, \mathbf{e}_{5}\right]=(\alpha-1) \mathbf{e}_{3},} & {\left[\mathbf{e}_{4}, \mathbf{e}_{5}\right]=\mathbf{e}_{4},} \\
\mathbf{2} &
\end{array}
$$


where all values of $\alpha \in \mathbb{R}$ are admissible. The isotropy is $\mathbf{h}=\operatorname{span}\left\{\mathbf{e}_{4}\right\}$.

A3 The Lie algebra $\mathbf{g}$ is one of the five-dimensional solvable algebras $A_{5,37}$ or $A_{5,36}$. There exits a basis for $\mathbf{g}$ where the non-zero products are

$\left[\mathbf{e}_{1}, \mathbf{e}_{4}\right]=2 \mathbf{e}_{1},\left[\mathbf{e}_{2}, \mathbf{e}_{3}\right]=\mathbf{e}_{1},\left[\mathbf{e}_{2}, \mathbf{e}_{4}\right]=\mathbf{e}_{2},\left[\mathbf{e}_{2}, \mathbf{e}_{5}\right]=-\epsilon \mathbf{e}_{3},\left[\mathbf{e}_{3}, \mathbf{e}_{4}\right]=\mathbf{e}_{3},\left[\mathbf{e}_{3}, \mathbf{e}_{5}\right]=\mathbf{e}_{2}$, where $\epsilon=1$ for $A_{5,37}$ and $\epsilon=-1$ for $A_{5,36}$. The isotropy is $\mathbf{h}=\operatorname{span}\left\{\mathbf{e}_{3}\right\}$.

A4 The Lie algebra $\mathbf{g}$ is the six-dimensional algebra $\mathbf{s l}(2, \mathbb{R}) \ltimes \mathbf{n}(3)$ where $\mathbf{n}(3)$ is the threedimensional Heisenberg algebra. There exits a basis for $\mathbf{g}$ where the non-zero products are

$$
\begin{array}{llll}
{\left[\mathbf{e}_{1}, \mathbf{e}_{2}\right]=2 \mathbf{e}_{2},} & {\left[\mathbf{e}_{1}, \mathbf{e}_{3}\right]=-2 \mathbf{e}_{3},} & {\left[\mathbf{e}_{2}, \mathbf{e}_{3}\right]=\mathbf{e}_{1},} & {\left[\mathbf{e}_{1}, \mathbf{e}_{4}\right]=\mathbf{e}_{4},} \\
{\left[\mathbf{e}_{1}, \mathbf{e}_{5}\right]=-\mathbf{e}_{5},} & {\left[\mathbf{e}_{2}, \mathbf{e}_{5}\right]=\mathbf{e}_{4},} & {\left[\mathbf{e}_{3}, \mathbf{e}_{4}\right]=\mathbf{e}_{5},} & {\left[\mathbf{e}_{4}, \mathbf{e}_{5}\right]=\mathbf{e}_{6} .}
\end{array}
$$

The isotropy is $\mathbf{h}=\operatorname{span}\left\{\mathbf{e}_{3}+\mathbf{e}_{6} \mathbf{e}_{5}\right\}$. The algebra is sometimes called the Schroedinger algebra.

$\mathbf{A 5}^{*}$ The Lie algebra $\mathbf{g}$ is the seven-dimensional algebra $\mathbf{s l}(2, \mathbb{R}) \ltimes A_{4,9}^{1}$. There exists a basis for $\mathrm{g}$ where the non-zero products are

$$
\begin{aligned}
& {\left[\mathbf{e}_{1}, \mathbf{e}_{2}\right]=2 \mathbf{e}_{2}, \quad\left[\mathbf{e}_{1}, \mathbf{e}_{3}\right]=-2 \mathbf{e}_{3}, \quad\left[\mathbf{e}_{1}, \mathbf{e}_{5}\right]=-\mathbf{e}_{5}, \quad\left[\mathbf{e}_{1}, \mathbf{e}_{6}\right]=\mathbf{e}_{6}, \quad\left[\mathbf{e}_{2}, \mathbf{e}_{3}\right]=\mathbf{e}_{1},} \\
& {\left[\mathbf{e}_{2}, \mathbf{e}_{5}\right]=\mathbf{e}_{6}, \quad\left[\mathbf{e}_{3}, \mathbf{e}_{6}\right]=\mathbf{e}_{5}, \quad\left[\mathbf{e}_{4}, \mathbf{e}_{7}\right]=2 \mathbf{e}_{4}, \quad\left[\mathbf{e}_{5}, \mathbf{e}_{6}\right]=\mathbf{e}_{4}, \quad\left[\mathbf{e}_{5}, \mathbf{e}_{7}\right]=\mathbf{e}_{5} \quad\left[\mathbf{e}_{6}, \mathbf{e}_{7}\right]=\mathbf{e}_{6} .}
\end{aligned}
$$

The isotropy is $\mathbf{h}=\operatorname{span}\left\{\mathbf{e}_{1}+\mathbf{e}_{7}, \mathbf{e}_{3}-\mathbf{e}_{4}, \mathbf{e}_{5}\right\}$.

In the book Einstein Spaces [18], Petrov gave a fairly comprehensive list of the possible infinitesimal generators for the isometry algebras of a four-dimensional Lorentz manifold. The Lie algebras in A1 and A4 should appear on the list, but they don't.

We now list the possibilities when the signature is $(2,2)$.

Theorem 2.3. Let $(G / H, \eta)$ be a four-dimensional homogeneous pseudo-Riemannian manifold of signature (2,2) where $H$ is connected. If $G / H$ is not reductive, then the Lie algebra pair $(\mathbf{g}, \mathbf{h})$ is isomorphic to one in the following list.

A1-A3 The corresponding Lie algebra pairs in Theorem 2.2.

B1 The Lie algebra $\mathbf{g}$ is the five-dimensional algebra $\mathbf{s l}(2, \mathbb{R}) \ltimes \mathbb{R}^{2}$. There exists a basis for $\mathbf{g}$ where the non-zero products are

$$
\begin{aligned}
& {\left[\mathbf{e}_{1}, \mathbf{e}_{2}\right]=2 \mathbf{e}_{2}, \quad\left[\mathbf{e}_{1}, \mathbf{e}_{3}\right]=-2 \mathbf{e}_{2}, \quad\left[\mathbf{e}_{2}, \mathbf{e}_{3}\right]=\mathbf{e}_{1}, \quad\left[\mathbf{e}_{1}, \mathbf{e}_{4}\right]=\mathbf{e}_{4},} \\
& {\left[\mathbf{e}_{1}, \mathbf{e}_{5}\right]=-\mathbf{e}_{5}, \quad\left[\mathbf{e}_{2}, \mathbf{e}_{5}\right]=\mathbf{e}_{4}, \quad\left[\mathbf{e}_{3}, \mathbf{e}_{4}\right]=\mathbf{e}_{5} .}
\end{aligned}
$$

The isotropy is $\mathbf{h}=\operatorname{span}\left\{\mathbf{e}_{3}\right\}$.

B2 The Lie algebra $\mathbf{g}$ is the six-dimensional Schroedinger algebra $\mathbf{s l}(2, \mathbb{R}) \ltimes \mathbf{n}(3)$ in $\mathbf{A 4}$ of Theorem 2.2 and the isotropy $\mathbf{h}=\operatorname{span}\left\{\mathbf{e}_{3}-\mathbf{e}_{6}, \mathbf{e}_{5}\right\}$.

B3 The Lie algebra $\mathbf{g}$ is the six-dimensional algebra $\mathbf{s l}(2, \mathbb{R}) \ltimes \mathbb{R}^{2} \oplus \mathbb{R}$. There exits a basis for $\mathrm{g}$ where the non-zero products are

$$
\begin{array}{lll}
{\left[\mathbf{e}_{1}, \mathbf{e}_{2}\right]=2 \mathbf{e}_{2},} & {\left[\mathbf{e}_{1}, \mathbf{e}_{3}\right]=-2 \mathbf{e}_{3},} & {\left[\mathbf{e}_{2}, \mathbf{e}_{3}\right]=\mathbf{e}_{1}, \quad\left[\mathbf{e}_{1}, \mathbf{e}_{4}\right]=\mathbf{e}_{4},} \\
{\left[\mathbf{e}_{1}, \mathbf{e}_{5}\right]=-\mathbf{e}_{5},} & {\left[\mathbf{e}_{2}, \mathbf{e}_{5}\right]=\mathbf{e}_{4},} & {\left[\mathbf{e}_{3}, \mathbf{e}_{4}\right]=\mathbf{e}_{5} .}
\end{array}
$$

The isotropy is $\mathbf{h}=\operatorname{span}\left\{\mathbf{e}_{3}, \mathbf{e}_{5}+\mathbf{e}_{6}\right\}$.

The following theorem proved in section 6 , gives a complete classification when the space is simply connected. 
Theorem 2.4. Let $G / H$ be a simply connected non-reductive pseudo-Riemannian homogeneous space of dimension four, then

i] $G / H$ is diffeomorphic to $\mathbb{R}^{4}$, and

ii] if $G$ is the full isometry group then the Lie algebra pair for $G / H$ is equivalent to one in Theorem 2.2 excluding $\mathbf{A 5} \mathbf{5}^{*}$, or to one in Theorem 2.3.

Conversely, for any Lie algebra pair from Theorem 2.2 except $\mathbf{A 5} \mathbf{5}^{*}$, or any in Theorem 2.3, there exists a pseudo-Riemannian metric on $\mathbb{R}^{4}$ (subject to the conditions on the signature), where the isometry group acts transitively on $\mathbb{R}^{4}$, the Lie algebra of the isometry group is the given Lie algebra $\mathbf{g}$, and the Lie algebra of the isotropy at a point is (conjugate to) $\mathbf{h}$.

We show in Lemma 5.1 that only A2 in Theorem 2.2 or 2.3 with $\alpha=\frac{2}{3}$ and B3 lead to Einstein spaces which are not of constant curvature. Furthermore, B3 is Ricci-flat. By using this result we prove in section 7 the following theorem which gives complete list of all the homogeneous Einstein metrics which are not of constant curvature for the simply connected non-reductive pseudo-Riemannian manifolds of dimension 4 .

Theorem 2.5. Let $(G / H, \eta)$ be a simply connected non-reductive homogeneous space of dimension 4 which is Einstein and not of constant curvature. If $\eta$ is Ricci-flat, then the Lie algebra pair is isomorphic to $\mathbf{B} 3$ and there exists global coordinates $\left(y^{i}\right)_{i=1 . .4}$ on $G / H=\mathbb{R}^{4}$ such that the metric is

$$
\eta=2 e^{y^{1}} \cos y^{2}\left(d y^{1} d y^{4}-d y^{2} d y^{3}\right)-2 e^{y^{1}} \sin y^{2}\left(d y^{1} d y^{3}+d y^{2} d y^{4}\right)+L e^{4 y^{1}}\left(d y^{2}\right)^{2}
$$

for some $L \in \mathbb{R}^{*}$. Otherwise the Lie algebra pair is isomorphic to $\mathbf{A 2}$ with $\alpha=2 / 3$ and there exists global coordinates $\left(y^{i}\right)_{i=1 . .4}$ on $G / H=\mathbb{R}^{4}$ such that the metric is

$$
\eta=a_{1} e^{-\frac{4}{3} y^{4}}\left(2 d y^{1} d y^{3}-\left(d y^{2}\right)^{2}\right)+a_{2} e^{\frac{2}{3} y^{4}}\left(d y^{3}\right)^{2}+2 a_{3} e^{\frac{1}{3} y^{4}} d y^{3} d y^{4}+a_{4}\left(d y^{4}\right)^{2}
$$

for some choice of $a_{i} \in \mathbb{R}, i=1 . .4$ where $a_{1} a_{4} \neq 0$, and $a_{2} \neq 0$.

It is worth noting that determining the Lie algebra of the isometry group for the Ricci flat metrics in this theorem is non-trivial.

\section{Cartan's Approach to the Geometry of Homogeneous Spaces}

Let $\eta^{0}$ be a non-degenerate symmetric bilinear form on $\mathbb{R}^{n}$ with signature $(p, \tilde{p})$, and $O(p, \tilde{p}) \subset$ $G L(n, \mathbb{R})$ be the Lie group preserving $\eta^{0}$. Let $(M, \eta)$ be a pseudo-Riemannian manifold of signature $(p, \tilde{p})$, and $\pi: O(M) \rightarrow M$ be the orthonormal frame bundle corresponding to $\eta^{0}$ defined by

$$
O(M)=\left\{u_{p}: \mathbb{R}^{n} \rightarrow T_{p} M \mid \eta\left(u_{p}(X), u_{p}(Y)\right)=\eta^{0}(X, Y)\right\} .
$$

Denote the right action of $a \in O(p, \tilde{p})$ on $u \in O(M)$ by $u a$, and for $X \in \mathbf{o}(p, \tilde{p})$, let $\tilde{X}$ be the corresponding infinitesimal generator on $O(M)$ defined by

$$
\tilde{X}_{u}=\left.\frac{d}{d t}\left(u \exp \left(t X_{e}\right)\right)\right|_{t=0} .
$$

The canonical $\mathbb{R}^{n}$-valued one-form $\theta$ and the $\mathbf{o}(p, \tilde{p})$-valued connection one-form $\omega$ on $O(M)$ have the following properties [9] (pp. 118-121)

$$
\iota_{Z} \theta=u^{-1} \pi_{*}(Z), \quad \iota_{\tilde{X}} \omega=X, \quad d \theta=-\omega \wedge \theta,
$$


where $X \in \mathbf{o}(p, \tilde{p}), Z \in T_{u}(O(M))$ and $\iota$ is left interior multiplication. The $\mathbf{o}(p, \tilde{p})$-valued curvature two-form $\Omega=d \omega+\omega \wedge$ satisfies

$$
\iota_{\tilde{X}} \Omega=0, \quad \Omega \wedge \theta=0, \quad d \Omega=\Omega \wedge \omega-\omega \wedge \Omega .
$$

The forms $\theta, \omega$, and $\Omega$ satisfy the equivariance conditions

$$
a^{*} \theta=a^{-1}(\theta), \quad a^{*} \omega=A d\left(a^{-1}\right) \omega, \quad a^{*} \Omega=A d\left(a^{-1}\right) \Omega \quad \text { where } a \in O(p, \tilde{p}) .
$$

If $H$ is connected, then (3.1) and (3.2) imply the equivariance of $\theta, \omega$ and $\Omega$.

Let $g: M \rightarrow M$ be an isometry of the pseudo-Riemannian manifold $(M, \eta)$, and $\phi_{g}$ be the lift of the diffeomorphism $g$ of $M$ to the frame bundle,

$$
\phi_{g}(u)=g_{*} u, \quad u \in F(M) .
$$

Since $g$ is an isometry, the subset $O(M) \subset F(M)$ is invariant under $\phi_{g}$. The forms $\theta, \omega$, and $\Omega$ satisfy the invariance properties

$$
\phi_{g}^{*} \theta=\theta, \phi_{g}^{*} \omega=\omega, \phi_{g}^{*} \Omega=\Omega
$$

Suppose that $(G / H, \eta)$ is a homogeneous pseudo-Riemannian manifold and let $\sigma=[H] \in G / H$, and $u_{\sigma} \in O(G / H)$ be an orthonormal frame at $\sigma$. The linear isotropy representation $\rho: H \rightarrow O(p, \tilde{p})$ is defined by

$$
u_{\sigma} \rho(h)=\left(L_{h}\right)_{*} u_{\sigma}
$$

where $L_{h}$ is right multiplication in $G$ by $h \in H$. The differential of $\rho$ defines a homomorphism $\rho_{*}: \mathbf{h} \rightarrow \mathbf{o}(p, \tilde{p})$. Since $G$ acts effectively and by isometries, the linear isotropy representation of $H$ is faithful. Following Cartan [3] (or see Jensen [8]), we define the function $\Psi: G \rightarrow O(G / H)$ by

$$
\Psi(g)=g_{*} u_{\sigma}
$$

which makes the diagram

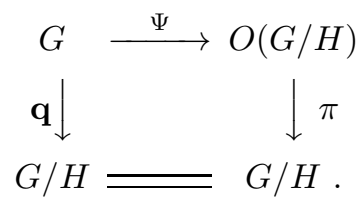

commutative. The map $\Psi$ is equivariant with respect to the left action of $G$ on $G$ and the action of $G$ on $O(M)$. It is also equivariant with respect to the linear isotropy representation. Therefore, $\Psi$ satisfies

$$
\Psi(g h)=\Psi(g) \rho(h), \quad \text { and } \quad \Psi\left(g_{1} g_{2}\right)=\phi_{g_{1}} \circ \Psi\left(g_{2}\right) .
$$

By defining the forms

$$
\hat{\theta}=\Psi^{*} \theta, \quad \hat{\omega}=\Psi^{*} \omega, \quad \hat{\Omega}=\psi^{*} \Omega
$$

which are $G$-invariant on account of the equivariance of $\Psi$ and equation (3.3), we obtain the following structure on the Lie algebra $\mathbf{g}$ of $G$. 
Lemma 3.1. Let $(G / H, \eta)$ be an n-dimensional homogeneous pseudo-Riemannian manifold with Lie algebra pair $(\mathbf{g}, \mathbf{h})$. There exists an injective homomorphism $\rho_{*}: \mathbf{h} \rightarrow \mathbf{o}(p, \tilde{p})$, an $\mathbb{R}^{n}$-valued one-form $\hat{\theta}: \mathbf{g} \rightarrow \mathbb{R}^{n}$, and an $\mathbf{o}(p, \tilde{p})$-valued one-form $\hat{\omega}: \mathbf{g} \rightarrow \mathbf{o}(p, \tilde{p})$ satisfying

1] $\operatorname{ker} \hat{\theta}=\mathbf{h}$,

2] $\hat{\omega}(X)=\rho_{*}(X)$, and

3] $d \hat{\theta}=-\hat{\omega} \wedge \hat{\theta}$

where $X \in \mathbf{h}$. Furthermore, the $\mathbf{o}(p, \tilde{p})$-valued two-form $\Omega=d \hat{\omega}+\hat{\omega} \wedge \hat{\omega}$ satisfies

4] $\iota_{X} \Omega=0, \quad \hat{\Omega} \wedge \hat{\theta}=0$, and $\quad d \hat{\Omega}=\hat{\Omega} \wedge \hat{\omega}-\hat{\omega} \wedge \hat{\Omega}$.

Lemma 3.1 has the following partial converse.

Lemma 3.2. Let $\mathbf{h}$ be a Lie algebra, $\rho_{*}: \mathbf{h} \rightarrow \mathbf{o}(p, \tilde{p})$ a monomorphism, and $\mathbf{g}$ be the vector space $\mathbb{R}^{n} \oplus \mathbf{h}$. Suppose there exists forms $\theta: \mathbf{g} \rightarrow \mathbb{R}^{n}, \omega: \mathbf{g} \rightarrow \mathbf{o}(p, \tilde{p})$, and $\Omega: \mathbf{g} \wedge \mathbf{g} \rightarrow \mathbf{o}(p, \tilde{p})$ satisfying

1] $\operatorname{ker} \theta=\mathbf{h}$,

$\hat{2}] \omega(X)=\rho_{*}(X)$,

$\hat{3}] \iota_{X} \Omega=0$, and $\Omega \wedge \theta=0$.

If we define $d \theta=-\omega \wedge \theta$ and $\Omega$ satisfies

4] $d \Omega=\Omega \wedge \omega-\omega \wedge \Omega$,

then with $d \omega=\Omega-\omega \wedge \omega, \mathbf{g}$ is a Lie algebra where $\alpha([X, Y])=-d \alpha(X, Y), \alpha \in \mathbf{g}^{*}, X, Y \in \mathbf{g}$.

The principle step in Cartan's approach to the classification of homogeneous pseudo-Riemannian manifolds is to start with a subalgebra $\mathbf{h} \subset \mathbf{o}(p, \tilde{p})$ and then classify all Lie algebras that satisfy Lemma 3.2. To simplify this classification, one expects that we only need the conjugacy class of the subalgebra $\mathbf{h} \subset \mathbf{o}(p, \tilde{p})$ under inner automorphism, but slightly more is true.

Lemma 3.3. Let $\mathbf{h}$ and $\tilde{\mathbf{h}}$ be two Lie algebras, and let $\rho_{*}: \mathbf{h} \rightarrow \mathbf{o}(p, \tilde{p})$ and $\tilde{\rho}_{*}: \tilde{\mathbf{h}} \rightarrow \mathbf{o}(p, \tilde{p})$ be monomorphisms. Suppose there exists an inner automorphism $\psi: \operatorname{gl}(n, \mathbb{R}) \rightarrow \operatorname{gl}(n, \mathbb{R})$ which restricts to an isomorphism $\phi: \mathbf{h} \rightarrow \tilde{\mathbf{h}}$ such that

$$
\tilde{\rho}_{*}(\phi(X))=\psi\left(\rho_{*}(X)\right) \quad X \in \mathbf{h} .
$$

Then the pairs $(\mathbf{g}, \mathbf{h})$ which satisfy Lemma 3.2 are in one to one correspondence with the pairs $(\tilde{\mathbf{g}}, \tilde{\mathbf{h}})$ which satisfy Lemma 3.2.

Proof. Suppose the inner automorphism $\psi$ is conjugation by the matrix $A \in G L(n, \mathbb{R})$. It is then easy to check that the vector-space isomorphism $T: \mathbb{R}^{n} \oplus \mathbf{h} \rightarrow \mathbb{R} \oplus \tilde{\mathbf{h}}$ defined by

$$
T(\xi, X)=(A \xi, \phi(X))
$$

provides a correspondence.

Note that every inner automorphism of $\mathbf{o}(p, \tilde{p})$ satisfies this lemma. 


\section{Non-Reductive Homogeneous Spaces}

The preceding section described Cartan's procedure for constructing all possible isomorphism classes of Lie algebra pairs $(\mathbf{g}, \mathbf{h})$ for homogeneous pseudo-Riemannian manifolds by starting from the inequivalent subalgebras of $\mathbf{o}(p, \tilde{p})$ under the automorphisms described in Lemma 3.3. In principle, one could find a general classification of the four-dimensional homogeneous pseudo-Riemannian manifolds starting with the entire list of subalgebras for the Lie algebras $\mathbf{o}(3,1)$ and $\mathbf{o}(2,2)$. This classification would be rather daunting because the known lists of inequivalent subalgebras under inner automorphisms are quite large [15], [16]. In this section, we simplify the classification problem by proving a lemma which reduces the possible subalgebras $\mathbf{h} \subset \mathbf{o}(p, \tilde{p})$ associated with a nonreductive homogeneous pseudo-Riemannian manifold $(G / H, \eta)$.

We start with the following characterization of reductive homogeneous spaces see [9], p. 103, Theorem 11.1.

Lemma 4.1. A homogeneous space $G \rightarrow G / H$ is reductive if and only if the principal $H$-bundle $G \rightarrow G / H$ admits a $G$-invariant connection.

The following lemma greatly simplifies the classification problem.

Lemma 4.2. If $G / H$ is a pseudo-Riemannian homogeneous space and $O(p, \tilde{p}) / \rho(H)$ is a reductive homogeneous space, then $G / H$ is reductive.

Proof. Let $\tilde{\mathbf{h}}$ be the Lie algebra of $\rho(H)$, and $\mathbf{o}(p, \tilde{p})=\tilde{\mathbf{h}} \oplus \mathbf{m}$ be a reductive decomposition of $\mathbf{o}(p, \tilde{p})$. Decompose the connection form on $O(G / H)$ as $\omega=\omega_{\tilde{\mathbf{h}}}+\omega_{\mathbf{m}}$ where $\omega_{\tilde{\mathbf{h}}}$ takes values in $\tilde{\mathbf{h}}$ and $\omega_{\mathbf{m}}$ takes values in $\mathbf{m}$. By using the map $\rho$ defined in (3.4) and $\Psi$ defined in (3.5) we prove that the h-valued form $\rho_{*}^{-1} \circ\left(\Psi^{*} \omega_{\tilde{\mathbf{h}}}\right)$ defines a $G$-invariant connection on $G / H$.

The $G$-invariance of $\rho_{*}^{-1} \circ\left(\Psi^{*} \omega_{\tilde{\mathbf{h}}}\right)$ follows from the equivariance of $\Psi$ in (3.7) together with (3.3). In order that this form defines a connection we need to check that the two conditions on p.64 in [9] are satisfied. To check the first condition on p.64 [9], we use (3.1) and compute

$$
\rho_{*}^{-1} \circ\left(\Psi^{*} \omega_{\tilde{\mathbf{h}}}\left(X_{e}\right)\right)=\rho_{*}^{-1} \circ \omega_{\tilde{\mathbf{h}}}\left(\rho_{*}\left(X_{e}\right)\right)=X_{e} .
$$

This verifies condition one. We now check the second condition. It follows from the hypothesis in the lemma and the equivariance of the connection form $\omega$ that

$$
R_{a}^{*} \omega_{\tilde{\mathbf{h}}}=A d_{a^{-1}} \omega_{\tilde{\mathbf{h}}} \quad \text { and } \quad R_{a}^{*} \omega_{\mathbf{m}}=A d_{a^{-1}} \omega_{\mathbf{m}}
$$

Now from the $H$-equivariance of $\Psi$ in (3.7), the equation above, and the identity $\rho_{*}^{-1} \circ A d_{\rho(h)}=$ $A d_{h} \circ \rho_{*}^{-1}$ it follows that

$$
R_{h}^{*}\left(\rho_{*}^{-1} \circ\left(\Psi^{*} \omega_{\tilde{\mathbf{h}}}\right)\right)=\rho_{*}^{-1} \circ\left(\Psi^{*} \rho(h)^{*} \omega_{\tilde{\mathbf{h}}}\right)=\rho_{*}^{-1} \circ\left(A d_{\rho(h)^{-1}} \circ \Psi^{*} \omega_{\tilde{\mathbf{h}}}\right)=A d_{h^{-1}} \circ \rho_{*}^{-1} \circ\left(\Psi^{*} \omega_{\tilde{\mathbf{h}}}\right) .
$$

This verifies the second condition. Therefore, the $\mathbf{h}$-valued form $\rho_{*}^{-1} \circ \Psi^{*} \omega_{\tilde{\mathbf{h}}}$, is a $G$-invariant connection on $G / H$ and by Lemma $4.1, G / H$ is reductive.

The proof of Lemma 4.2 is similar to the proof of Proposition 6.4 on pg. 83 in [9]. This proposition implies that if $O(p, \tilde{p}) / H$ is reductive, then the metric connection is reducible to $H$.

This lemma has a few simple but interesting corollaries.

Corollary 4.1. If $\rho_{*}(\mathbf{h}) \subset \mathbf{o}(p, \tilde{p})$ is a non-degenerate subspace with respect to the Killing form of $\mathbf{o}(p, \tilde{p})$, then $G / H$ is reductive.

Lemma 4.2 also provides an algebraic proof of the following corollaries. 
Corollary 4.2. If $G / H$ admits a $G$ invariant Riemannian metric, then $G / H$ is a reductive homogeneous space.

Corollary 4.3. If $(G / H, \eta)$ is a two dimension homogeneous Lorentz manifold, then $G / H$ is reductive.

\section{The Computations .}

By starting with the inequivalent subalgebras of $\mathbf{o}(2,1), \mathbf{o}(3,1)$, and $\mathbf{o}(2,2)$ we prove Theorems 2.1, 2.2 and 2.3 by building all non-reductive Lie algebra pairs $(\mathbf{g}, \mathbf{h})$ which satisfy Lemma 3.2. All inequivalent subalgebras of $\mathbf{o}(2,1), \mathbf{o}(3,1), \mathbf{o}(2,2)$ under inner automorphisms are known. Although this list is rather long, Lemma 4.2 says that we need only those subalgebras that are not reductive in their respective algebras. With this reduced list of subalgebras, the equivalence problem in Lemma 3.3 is much easier. By using this final list of inequivalent subalgebras, we determine those which extend to a Lie algebra that satisfies Lemma 3.2 and not Lemma 4.1 (see also Lemma A1). The resulting Lie algebra pairs are then put into a canonical form which proves Theorems 2.1, 2.2 and 2.3 .

Let $\left(e_{j}^{i}\right)$ denote the standard basis for $g l(n, \mathbb{R})$ where

$$
\left(e_{j}^{i}\right)_{l}^{k}=\delta_{k}^{i} \delta_{j}^{l}, \quad 1 \leq i, j, k, l \leq n .
$$

Hereafter we omit writing the isomorphism $\rho_{*}$ between $\mathbf{h}$ with basis $\left\{\mathbf{e}_{\alpha}\right\}_{\alpha=1 \ldots q}$ and $\rho_{*}(\mathbf{h}) \subset \mathbf{o}(p, \tilde{p})$ with basis $\left\{\mathbf{b}_{\alpha}\right\}_{\alpha=1 \ldots q}$. Given two differential one forms $\sigma^{1}, \sigma^{2} \in \Omega^{1}(M)$, we use the convention

$$
\sigma^{1} \sigma^{2}=\frac{1}{2}\left(\sigma^{1} \otimes \sigma^{2}+\sigma^{2} \otimes \sigma^{1}\right)
$$

for the symmetric tensor product. Other notation that is used is given in Appendix A.

Proof of Theorem 2.1-o $(2,1)$ : Let $\left\{\sigma^{i}\right\}_{i=1 \ldots 3}$ denote the standard basis for $\left(\mathbb{R}^{3}\right)^{*}$, and

$$
\eta^{0}=\left(\sigma^{1}\right)^{2}+2 \sigma^{2} \sigma^{3}
$$

For $\mathbf{o}(2,1)$ we use the basis

$$
B_{1}=e_{2}^{2}-e_{3}^{3}, B_{2}=e_{2}^{1}-e_{1}^{3}, B_{3}=e_{1}^{2}-e_{3}^{1} .
$$

Of the inequivalent subalgebras of $\mathbf{o}(2,1)$ under inner automorphism, only two are not reductive. In each case, by using equations (A.1) and (A.3), equation (A.5) always has a solution, so for these two subalgebras, the constructed homogeneous space will be reductive. Here are the details.

Case 1: The isotropy subalgebra is $\mathbf{h}=\operatorname{span}\left\{\mathbf{b}_{1}=B_{3}\right\}$. By using the basis $\left\{\tilde{\mathbf{b}}_{1}=B_{1}, \tilde{\mathbf{b}}_{2}=B_{2}, \mathbf{b}_{1}\right\}$ for $\mathbf{o}(2,1)$, equations (A.1) and (A.3) give

$$
\tilde{\omega}^{1}=p_{1} \theta^{1}+p_{2} \theta^{3}, \quad \tilde{\omega}^{2}=-p_{1} \theta^{3} .
$$

Equation (A.5) has the general solution

$$
r_{1}^{1}=-p_{2}, \quad r_{2}^{1}=p_{1},
$$

and $\left(\omega^{1}-p_{2} \theta^{1}+p_{1} \theta^{2}\right) \otimes \mathbf{e}_{1}$ defines a $G$-invariant connection. 
Case 2: The isotropy subalgebra is $\mathbf{h}=\operatorname{span}\left\{\mathbf{b}_{1}=B_{1}, \mathbf{b}_{2}=B_{3}\right\}$. By using the basis $\left\{\tilde{\mathbf{b}}_{1}=\right.$ $\left.B_{2}, \mathbf{b}_{1}, \mathbf{b}_{2}\right\}$ for $\mathbf{o}(2,1)$, equations (A.1) and (A.3) give

$$
\tilde{\omega}^{1}=p_{1} \theta^{3} \text {. }
$$

Equation (A.5) has the general solution

$$
r_{1}^{1}=p_{1}, \quad r_{2}^{1}=r_{3}^{1}=r_{1}^{2}=r_{3}^{2}=0, \quad r_{2}^{2}=-p_{1},
$$

and $\left(\omega^{1}+p_{1} \theta^{1}\right) \otimes \mathbf{e}_{1}+\left(\omega^{2}-p_{1} \theta^{2}\right) \otimes \mathbf{e}_{2}$ defines a $G$-invariant connection.

This proves Theorem 2.1 .

Proof of Theorem 2.2 - o(3,1): Let $\left\{\sigma^{i}\right\}_{i=1 \ldots 4}$ denote the standard basis for $\left(\mathbb{R}^{4}\right)^{*}$, and

$$
\eta^{0}=\left(\sigma^{1}\right)^{2}+\left(\sigma^{2}\right)^{2}+2 \sigma^{3} \sigma^{4}
$$

The basis for $\mathbf{o}(3,1)$ we use is

$$
B_{1}=e_{1}^{2}-e_{2}^{1}, \quad B_{2}=e_{4}^{4}-e_{3}^{3}, \quad B_{3}=e_{1}^{4}-e_{3}^{1}, \quad B_{4}=e_{2}^{4}-e_{3}^{2}, \quad B_{5}=e_{4}^{1}-e_{1}^{3}, \quad B_{6}=e_{2}^{3}-e_{4}^{2} .
$$

On page 1605 of [15], the inequivalent subalgebras of $\mathbf{o}(3,1)$, under inner automorphisms are listed. Of these subalgebras, labeled $F_{1}$ to $F_{15}$, seven are not reductive in $\mathbf{o}(3,1)$.

Case 1: We consider the non-reductive subalgebras of $\mathbf{o}(3,1)$ which admit a solution to equation (A.5). Therefore they always lead to a reductive homogeneous space.

Subcase 1.1: The subalgebra $F_{2}$ in [15] is $\mathbf{h}=\operatorname{span}\left\{\mathbf{b}_{1}=B_{1}, \mathbf{b}_{2}=B_{2}, \mathbf{b}_{3}=B_{3}, \mathbf{b}_{4}=B_{4}\right\}$. By using the basis $\left\{\tilde{\mathbf{b}}_{1}=B_{5}, \tilde{\mathbf{b}}_{2}=B_{6}, \mathbf{b}_{1}, \mathbf{b}_{2}, \mathbf{b}_{3}, \mathbf{b}_{4}\right\}$ for $\mathbf{o}(3,1)$, equations (A.1) and (A.3) give

$$
\tilde{\omega}^{1}=0, \quad \tilde{\omega}^{2}=0 .
$$

Equation (A.5) has the general solution $\left\{r_{i}^{\alpha}=0\right\}_{\alpha=1 \ldots 2, i=1 \ldots 4}$, and $\omega^{\alpha} \otimes \mathbf{e}_{\alpha}$ defines a $G$-invariant connection.

Subcase 1.2: The subalgebra $F_{5}$ in [15] is $\mathbf{h}=\operatorname{span}\left\{\mathbf{b}_{1}=\cos \theta B_{1}+\sin \theta B_{2}, \mathbf{b}_{2}=B_{3}, \mathbf{b}_{3}=\right.$ $\left.B_{4}\right\}$ where $\theta \in(0, \pi), \theta \neq \pi / 2$. By using the basis $\left\{\tilde{\mathbf{b}}_{1}=-\sin \theta B_{1}+\cos \theta B_{2}, \tilde{\mathbf{b}}_{2}=B_{5}, \tilde{\mathbf{b}}_{3}=\right.$ $\left.B_{6}, \mathbf{b}_{1}, \mathbf{b}_{2}, \mathbf{b}_{3}\right\}$ for $\mathbf{o}(3,1)$, equations (A.1) and (A.3) give

$$
\tilde{\omega}^{1}=0, \quad \tilde{\omega}^{2}=0, \quad \tilde{\omega}^{3}=0 .
$$

Equation (A.5) has the general solution $\left\{r_{i}^{\alpha}=0\right\}_{\alpha=1 \ldots 2, i=1 \ldots 4}$ and $\omega^{\alpha} \otimes \mathbf{e}_{\alpha}$ defines a $G$-invariant connection.

Subcase 1.3: The subalgebra $F_{6}$ in $[15]$ is $\mathbf{h}=\operatorname{span}\left\{\mathbf{b}_{1}=B_{1}, \mathbf{b}_{2}=B_{3}, \mathbf{b}_{3}=B_{4}\right\}$. By using the basis $\left\{\tilde{\mathbf{b}}_{1}=B_{2}, \tilde{\mathbf{b}}_{2}=B_{5}, \tilde{\mathbf{b}}_{3}=B_{6}, \mathbf{b}_{1}, \mathbf{b}_{2}, \mathbf{b}_{3}\right\}$ for $\mathbf{o}(3,1)$, equations (A.1) and (A.3) give

$$
\tilde{\omega}^{1}=p_{1} \theta^{3}, \quad \tilde{\omega}^{2}=0, \quad \tilde{\omega}^{3}=0 .
$$

Equation (A.5) has the general solution

$$
r_{3}^{1}=r_{1}^{3}=t, \quad r_{2}^{2}=-t, \quad r_{1}^{1}=r_{2}^{1}=r_{4}^{1}=\underset{9}{r_{3}^{2}}=r_{4}^{2}=r_{3}^{3}=r_{4}^{3}=0, \quad r_{1}^{2}=r_{2}^{3}=-p_{1}
$$


where $t \in \mathbb{R}$, and $\left(\omega^{1}+t \theta^{3}\right) \otimes \mathbf{e}_{1}+\left(\omega^{2}-p_{1} \theta^{1}-t \theta^{2}\right) \otimes \mathbf{e}_{2}+\left(\omega^{3}+t \theta^{1}-p_{1} \theta^{2}\right) \otimes \mathbf{e}_{3}$ defines a $G$-invariant connection for any choice of $t \in \mathbb{R}$.

Subcase 1.4: The subalgebra $F_{8}$ in [15] is $\mathbf{h}=\operatorname{span}\left\{\mathbf{b}_{1}=B_{2}, \mathbf{b}_{2}=B_{3}\right\}$. By using the basis $\left\{\tilde{\mathbf{b}}_{1}=B_{1}, \tilde{\mathbf{b}}_{2}=B_{4}, \tilde{\mathbf{b}}_{3}=B_{5}, \tilde{\mathbf{b}}_{4}=B_{6}, \mathbf{b}_{1}, \mathbf{b}_{2}\right\}$ for $\mathbf{o}(3,1)$, equations (A.1) and (A.3) give

$$
\tilde{\omega}^{1}=-p_{1} \theta^{1}, \quad \tilde{\omega}^{2}=p_{1} \theta^{4}, \quad \tilde{\omega}^{3}=p_{2} \theta^{3}, \quad \tilde{\omega}^{4}=p_{1} \theta^{3} .
$$

Equation (A.5) has the general solution

$$
r_{1}^{1}=p_{2}, \quad r_{1}^{2}=r_{2}^{1}=r_{2}^{2}=r_{3}^{1}=r_{3}^{2}=r_{4}^{1}=0, \quad r_{4}^{2}=-p_{2},
$$

and $\left(\omega^{1}+p_{2} \theta^{1}\right) \otimes \mathbf{e}_{1}+\left(\omega^{2}-p_{2} \theta^{4}\right) \otimes \mathbf{e}_{2}$ defines a $G$-invariant connection.

We now consider the cases where condition (A.5) is not automatically satisfied.

Case 2: The subalgebra $F_{7}$ in [15] is $\mathbf{h}=\operatorname{span}\left\{\mathbf{b}_{1}=B_{2}, \mathbf{b}_{2}=B_{3}, \mathbf{b}_{3}=B_{4}\right\}$. By using the basis $\left\{\tilde{\mathbf{b}}_{1}=B_{1}, \tilde{\mathbf{b}}_{2}=B_{5}, \tilde{\mathbf{b}}_{3}=B_{6}, \mathbf{b}_{1}, \mathbf{b}_{2}, \mathbf{b}_{3}\right\}$ for $\mathbf{o}(3,1)$, equations (A.1) and (A.3) give

$$
\tilde{\omega}^{1}=p_{1} \theta^{1}+p_{2} \theta^{2}, \quad \tilde{\omega}^{2}=-p_{2} \theta^{3}, \quad \tilde{\omega}^{3}=-p_{1} \theta^{3} .
$$

From condition (A.5), $G / H$ is reductive if and only if $p_{1}=p_{2}=0$, so we assume this is not satisfied. Let $K=p_{1}{ }^{2}+p_{2}{ }^{2}$ (which is non-zero). The Bianchi identities give

$$
C_{42}^{1}=-K, C_{43}^{2}=-K, C_{41}^{3}=-K,
$$

and $C_{j k}^{\alpha}=0$ otherwise. The curvature form is $\Omega_{i j}=-K \theta_{i} \wedge \theta_{j}$, and the homogeneous space will be of constant curvature. The change of basis

$\alpha^{1}=\left(p_{1} \theta^{2}-p_{2} \theta^{1}-\omega^{1}\right) / 2, \alpha^{3}=\theta^{4}+K^{-1}\left(p_{1} \omega^{3}-p_{2} \omega^{2}\right), \alpha^{4}=\theta^{4}+K^{-1}\left(p_{2} \omega^{2}-p_{1} \omega^{3}\right), \alpha^{2}=K \theta^{3}$, $\alpha^{5}=\sqrt{2} K^{-1}\left(p_{1} \omega^{2}+p_{2} \omega^{3}\right), \alpha^{6}=\sqrt{2}\left(p_{1} \theta^{1}+p_{2} \theta^{2}\right), \quad \alpha^{7}=\left(p_{2} \theta^{1}-p_{1} \theta^{2}-\omega^{1}\right) / 2$

for $\mathbf{g}^{*}$ leads to the multiplication table $\mathbf{A} \mathbf{5}^{*}$ in Theorem 2.2 with isotropy in the dual basis $\mathbf{h}=$ $\operatorname{span}\left\{\mathbf{e}_{1}+\mathbf{e}_{7}, \mathbf{e}_{3}-\mathbf{e}_{4}, \mathbf{e}_{5}\right\}$.

Case 3: The subalgebra $F_{10}$ in $[15]$ is $\mathbf{h}=\operatorname{span}\left\{\mathbf{b}_{1}=B_{3}, \mathbf{b}_{2}=B_{4}\right\}$. By using the basis $\left\{\tilde{\mathbf{b}}_{1}=\right.$ $\left.B_{1}, \tilde{\mathbf{b}}_{2}=B_{2}, \tilde{\mathbf{b}}_{3}=B_{5}, \tilde{\mathbf{b}}_{4}=B_{6}, \mathbf{b}_{1}, \mathbf{b}_{2}\right\}$ for $\mathbf{o}(3,1)$, equations (A.1) and (A.3) give

$$
\tilde{\omega}^{1}=p_{1} \theta^{1}+p_{2} \theta^{2}+p_{3} \theta^{3}, \quad \tilde{\omega}^{2}=p_{2} \theta^{1}-p_{1} \theta^{2}+p_{4} \theta^{3}, \quad \tilde{\omega}^{3}=-p_{2} \theta^{3}, \quad \tilde{\omega}^{4}=-p_{1} \theta^{3} .
$$

From condition (A.5), $G / H$ is reductive if and only if $p_{1}=p_{2}=0$. Let $K=-\left(p_{1}{ }^{2}+p_{2}{ }^{2}\right)$ (which is non-zero) and $C_{13}^{1}=L\left({p_{1}}^{2}+4 p_{2}{ }^{2}\right)$. The Bianchi identities give

$C_{12}^{1}=-3 p_{2} p_{3}, C_{14}^{1}=-K, C_{34}^{1}=2 p_{4} p_{2}-p_{1} p_{3}, \quad C_{24}^{1}=C_{14}^{2}=0, \quad C_{23}^{1}=-3 L p_{2} p_{1}, p_{3}=0$, $C_{12}^{2}=3 p_{1} p_{3}, \quad C_{24}^{2}=-K, C_{34}^{2}=-2 p_{4} p_{1}-p_{2} p_{3}, C_{23}^{2}=\left(4 p_{1}^{2}+p_{2}^{2}\right) L, C_{13}^{2}=-3 L p_{2} p_{1}, p_{4}=0$.

The curvature components are

$$
\begin{aligned}
& \Omega_{12}=K \theta^{1} \wedge \theta^{2}, \Omega_{13}=K \theta^{1} \wedge \theta^{4}+L\left({p_{1}}^{2}+4 p_{2}{ }^{2}\right) \theta^{1} \wedge \theta^{3}-3 L p_{2} p_{1} \theta^{2} \wedge \theta^{3}, \Omega_{14}=K \theta^{1} \wedge \theta^{3}, \\
& \Omega_{24}=K \theta^{2} \wedge \theta^{3}, \Omega_{23}=K \theta^{2} \wedge \theta^{4}+L\left(4_{1}{ }^{2}+{p_{2}}^{2}\right) \theta^{2} \wedge \theta^{3}-3 L p_{2} p_{1} \theta^{1} \wedge \theta^{3}, \Omega_{34}=K \theta^{4} \wedge \theta^{3}
\end{aligned}
$$


The change of basis

$$
\begin{array}{lll}
\alpha^{1}=p_{1} \theta^{2}-p_{2} \theta^{1}, & \alpha^{2}=\theta^{3}, & \alpha^{3}=-K\left(\theta^{4}-L \theta^{3}\right)-p_{2} \omega^{1}+p_{1} \omega^{2} \\
\alpha^{4}=\sqrt{2}\left(p_{1} \theta^{1}+p_{2} \theta^{2}\right), & \alpha^{5}=\sqrt{2}\left(p_{1} \omega^{1}+p_{2} \omega^{2}\right), & \alpha^{6}=K\left(\theta^{4}+L \theta^{3}\right)-p_{2} \omega^{1}+p_{1} \omega^{2}
\end{array}
$$

for $\mathbf{g}^{*}$ leads to the multiplication table $\mathbf{A} 4$ in Theorem 2.2 with isotropy in the dual basis $\mathbf{h}=$ $\operatorname{span}\left\{\mathbf{e}_{3}+\mathbf{e}_{6}, \mathbf{e}_{5}\right\}$.

Case 4 The subalgebra $F_{14}$ in [15] is $\mathbf{h}=\operatorname{span}\left\{\mathbf{b}_{1}=B_{3}\right\}$. By using the basis $\left\{\tilde{\mathbf{b}}_{1}=B_{1}, \tilde{\mathbf{b}}_{2}=\right.$ $\left.B_{2}, \tilde{\mathbf{b}}_{3}=B_{4}, \tilde{\mathbf{b}}_{4}=B_{5}, \tilde{\mathbf{b}}_{5}=B_{6}, \mathbf{b}_{1}\right\}$ for $\mathbf{o}(3,1)$, equations (A.1) and (A.3) give

$$
\begin{aligned}
& \tilde{\omega}^{1}=p_{1} \theta^{1}+p_{2} \theta^{3}, \quad \tilde{\omega}^{2}=p_{3} \theta^{1}+p_{4} \theta^{2}+p_{5} \theta^{3}, \quad \tilde{\omega}^{3}=p_{2} \theta^{1}+p_{6} \theta^{2}+p_{7} \theta^{3}-p_{1} \theta^{4}, \\
& \tilde{\omega}^{4}=-p_{3} \theta^{3}, \quad \tilde{\omega}^{5}=-p_{1} \theta^{1} \text {. }
\end{aligned}
$$

From condition (A.5), $G / H$ is reductive if and only if $p_{4}=0$, so we assume $p_{4} \neq 0$. The first Bianchi identity gives

$C_{14}^{1}=-p_{3}{ }^{2}, C_{12}^{1}=-p_{2} p_{3}-p_{6} p_{1}, C_{24}^{1}=0, C_{23}^{1}=-3 p_{7} p_{3}+p_{2}\left(p_{5}-p_{6}\right), C_{34}^{1}=2 p_{2}\left(p_{1}+p_{4}\right)+2 p_{5} p_{3}$

and

$$
p_{6}=t_{1}\left(p_{1}-p_{4}\right), \quad p_{5}=t_{1}\left(p_{4}+p_{1}\right), \quad-p_{6} p_{3}+p_{2} p_{4}-2 p_{1} p_{2}=0, \quad p_{1} p_{3}=0
$$

where $t_{1} \in \mathbb{R}$. These last two equations will split into a number of cases. If $p_{3} \neq 0$ the Killing form will have rank 4, otherwise the Killing form has rank at most 3, so we split this case into subcases based on $p_{3}$.

Subcase 4.1 Starting with $p_{3} \neq 0$ and $p_{1}=0$, we solve (5.3) and the second Bianchi identity to get

$$
p_{2}=-t_{1} p_{3}, \quad C_{13}^{1}=t_{2} p_{3}^{2}, \quad p_{7}=-p_{4}\left(2 t_{1}^{2}+t_{2}\right) / 4
$$

where $t_{2} \in \mathbb{R}$. The curvature components are

$\Omega_{12}=K \theta^{1} \wedge \theta^{3}, \Omega_{13}=K \theta^{1} \wedge\left(\theta^{2}-t_{1} \theta^{3}\right)+p_{3}{ }^{2} \theta^{1} \wedge\left(t_{2} \theta^{3}-\theta^{4}\right)+\frac{3}{4} L p_{3} p_{4} \theta^{2} \wedge \theta^{3}, \Omega_{14}=-p_{3}{ }^{2} \theta^{1} \wedge \theta^{3}$,

$\Omega_{23}=K \theta^{3} \wedge\left(\theta^{4}-t_{1} \theta^{2}\right)+\frac{1}{2} p_{4} L\left(p_{4} \theta^{2}+\frac{3}{2} p_{3} \theta^{1}\right) \wedge \theta^{3}, \Omega_{24}=0, \Omega_{34}=K \theta^{2} \wedge \theta^{3}+p_{3}{ }^{2} \theta^{3} \wedge \theta^{4}$

where $K=t_{1} p_{3}{ }^{2}$ and $L=t_{2}-2 t_{1}{ }^{2}$. The change of basis

$$
\begin{aligned}
& \alpha^{1}=-p_{3} \theta^{1}-\frac{1}{2} p_{4} \theta^{4}, \quad \alpha^{2}=p_{3} \theta^{3}, \quad \alpha^{4}=-\frac{1}{2} t_{2} p_{3} \theta^{3}-p_{3} \theta^{4}-\omega^{1}, \quad \alpha^{5}=-p_{4} \theta^{2}-t_{1} p_{4} \theta^{3}, \\
& \alpha^{3}=t_{1} p_{4} \theta^{1}+p_{3} \theta^{4}+t_{1}\left(p_{4}{ }^{2}-2 p_{3}{ }^{2}\right)\left(2 p_{3}\right)^{-1} \theta^{2}+\left(t_{1}{ }^{2}\left(p_{4}{ }^{2}-2 p_{3}{ }^{2}\right)-t_{2} p_{3}{ }^{2}\right)\left(4 p_{3}\right)^{-1} \theta^{3}-\omega^{1}
\end{aligned}
$$

for $\mathbf{g}^{*}$ leads to the multiplication table $\mathbf{A} \mathbf{1}$ in Theorem 2.2 with isotropy in the dual basis $\mathbf{h}=$ $\operatorname{span}\left\{\mathbf{e}_{3}+\mathbf{e}_{4}\right\}$.

Subcase 4.2 Starting with $p_{3}=0$ the Bianchi identities give

$$
p_{2}=0, \quad\left(p_{1}-p_{4}\right)\left(C_{13}^{1}-t_{1}^{2} p_{1} p_{4}\right)=0 .
$$

The solution to this last equation splits into two further subcases. 
Subcase 4.2.a: If $C_{13}^{1}=t_{1}^{2} p_{1} p_{4}$ (the Killing form has rank 1), the curvature components are

$$
\begin{array}{lll}
\Omega_{12}=-p_{1}{ }^{2} \theta^{1} \wedge \theta^{2}, & \Omega_{13}=p_{1} L \theta^{1} \wedge \theta^{3}-p_{1}{ }^{2} \theta^{1} \wedge \theta^{4}, & \Omega_{14}=-p_{1}{ }^{2}, \\
\Omega_{24}=-p_{1}{ }^{2} \theta^{2} \wedge \theta^{3}, & \Omega_{23}=2 L\left(p_{1}-p_{4}\right) \theta^{2} \wedge \theta^{3}-p_{1}{ }^{2} \theta^{2} \wedge \theta^{4}, & \Omega_{34}=p_{1}{ }^{2} \theta^{3} \wedge \theta^{4}
\end{array}
$$

where $L=\left(p_{7}+t_{1}^{2} p_{4}\right)$. The change of basis

$$
\alpha^{1}=\theta^{4}-p_{7}\left(2 p_{4}\right)^{-1} \theta^{3}, \quad \alpha^{2}=-\theta^{2}, \quad \alpha^{3}=-\theta^{3}, \quad \alpha^{5}=\omega^{1}-t_{1} p_{1} \theta^{1}, \quad \alpha^{6}=-p_{4}\left(\theta^{2}+t_{1} \theta^{3}\right)
$$

for $\mathbf{g}^{*}$ leads to the multiplication table $\mathbf{A 2}$ in Theorem 2.2 where $\alpha=p_{1} / p_{4}$ and the isotropy in the dual basis is $\mathbf{h}=\operatorname{span}\left\{\mathbf{e}_{4}\right\}$.

Subcase 4.2.b We assume $C_{13}^{1}-t_{1}^{2} p_{1} p_{4} \neq 0$ so $p_{1}=p_{4}$, and the Killing form has rank 2. Let $L=C_{13}^{1}+p_{4} p_{7}$. The curvature components are

$$
\begin{array}{lll}
\Omega_{12}=-p_{4}{ }^{2} \theta^{1} \wedge \theta^{2}, & \Omega_{13}=L \theta^{1} \wedge \theta^{3}-p_{4}^{2} \theta^{1} \theta^{4}, & \Omega_{14}=-p_{4}{ }^{2} \theta^{1} \wedge \theta^{3}, \\
\Omega_{23}=-p_{4}{ }^{2} \theta^{2} \wedge \theta^{4}, & \Omega_{24}=p_{4}{ }^{2} \theta^{3} \wedge \theta^{4}, & \Omega_{34}=0 .
\end{array}
$$

Write $C_{13}^{1}-t_{1}^{2} p_{4}^{2}=\epsilon m^{2}$ (which is non-zero) where $\epsilon= \pm 1$. The change of basis

$$
\alpha^{1}=m \theta^{4}-m p_{7}\left(2 p_{4}\right)^{-1} \theta^{3}, \quad \alpha^{2}=m \theta^{1}, \quad \alpha^{3}=t_{1} p_{4} \theta^{1}-\omega^{1}, \quad \alpha^{4}=-p_{4} \theta^{2}-t_{1} p_{4} \theta^{3}, \quad \alpha^{5}=m \theta^{3}
$$

for $\mathbf{g}^{*}$ leads to the multiplication table $\mathbf{A 3}$ in Theorem 2.2 with isotropy in the dual basis $\mathbf{h}=$ $\operatorname{span}\left\{\mathbf{e}_{3}\right\}$.

This concludes the proof of Theorem 2.2.

Proof of Theorem 2.3- o(2,2): Let $\left\{\sigma^{i}\right\}_{i=1 \ldots 4}$ denote the standard basis for $\left(\mathbb{R}^{4}\right)^{*}$, and

$$
\eta^{0}=2 \sigma^{1} \sigma^{2}++2 \sigma^{3} \sigma^{4}
$$

For $\mathbf{o}(2,2)$ use the basis

$$
\begin{array}{lll}
2 A_{1}=e_{4}^{1}+e_{3}^{2}+e_{2}^{3}+e_{1}^{4}, & 2 A_{2}=e_{3}^{1}-e_{4}^{2}+e_{1}^{3}-e_{2}^{4}, & 2 A_{3}=e_{2}^{1}-e_{1}^{2}+e_{4}^{3}-e_{3}^{4} \\
2 B_{1}=-e_{4}^{1}+e_{3}^{2}+e_{2}^{3}-e_{1}^{4}, & 2 B_{2}=e_{3}^{1}+e_{4}^{2}+e_{1}^{3}+e_{2}^{4}, & 2 B_{3}=e_{2}^{1}-e_{1}^{2}-e_{4}^{3}+e_{3}^{4}
\end{array}
$$

On pages 2281-2283 of [16] the inequivalent subalgebras of $\mathbf{o}(3,1)$, under inner automorphisms are listed. Of these subalgebras, labeled $e_{d, n}$, twenty-two are not reductive in $\mathbf{o}(2,2)$.

Case 1: We consider the non-reductive subalgebras of $\mathbf{o}(2,2)$ which admit a solution to equation (A.5). Therefore, they always lead to a reductive homogeneous space.

Subcase 1.1: The subalgebra $e_{5,1}$ in [16] is $\mathbf{h}=\operatorname{span}\left\{\mathbf{b}_{1}=A_{2}, \mathbf{b}_{2}=A_{1}-A_{3}, \mathbf{b}_{3}=B_{1}, \mathbf{b}_{4}=\right.$ $\left.B_{2}, \mathbf{b}_{5}=B_{3}\right\}$. By using the basis $\left\{\tilde{\mathbf{b}}_{1}=A_{1}+A_{3}, \mathbf{b}_{1}, \mathbf{b}_{2}, \mathbf{b}_{3}, \mathbf{b}_{4}, \mathbf{b}_{5}\right\}$ for $\mathbf{o}(2,2)$, equations (A.1) and (A.3) give $\tilde{\omega}^{1}=0$. Equation (A.5) has the general solution $\left\{r_{k}^{\alpha}=0\right\}_{\alpha=1 \ldots 5, k=1 \ldots 4}$, and $\omega^{\alpha} \otimes \mathbf{e}_{\alpha}$ defines a $G$-invariant connection.

Subcase 1.2: The following 4-dimensional algebras in [16] always admit a solution to (A.5). Therefore, they always lead to the construction of a reductive homogeneous space. 


\begin{tabular}{|l|c|c|}
\hline $\mathbf{h}$ & Basis $\left\{\mathbf{b}_{1}, \mathbf{b}_{2}, \mathbf{b}_{3}, \mathbf{b}_{4}\right\}$ & Complement $\left\{\tilde{\mathbf{b}}_{1}, \tilde{\mathbf{b}}_{2}\right\}$ \\
\hline$e_{4,2}$ & $A_{1}-A_{3}, A_{2}, B_{2}, B_{1}-B_{3}$ & $A_{1}+A_{3}, B_{1}+B_{3}$ \\
$e_{4,3}$ & $A_{1}-A_{3}, B_{1}, B_{2}, B_{3}$ & $A_{1}+A_{3}, A_{2}$ \\
\hline
\end{tabular}

Equations (A.1) and (A.3) give $\tilde{\omega}^{1}=\tilde{\omega}^{2}=0$. Equation (A.5) has the general solution $\left\{r_{k}^{\alpha}=\right.$ $0\}_{\alpha=1 \ldots 4, k=1 \ldots 4}$, and $\omega^{\alpha} \otimes \mathbf{e}_{\alpha}$ defines a $G$-invariant connection.

Subcase 1.3: The following 3-dimensional algebras in [16] always admit a solution to (A.5). Therefore, they lead to the construction of a reductive homogeneous space.

\begin{tabular}{|l|c|c|}
\hline $\mathbf{h}$ & Basis $\left\{\mathbf{b}_{1}, \mathbf{b}_{2}, \mathbf{b}_{3}\right\}$ & Complement $\left\{\tilde{\mathbf{b}}_{1}, \tilde{\mathbf{b}}_{2} \tilde{\mathbf{b}}_{3}\right\}$ \\
\hline$e_{3,2}$ & $B_{2}, A_{2}, A_{1}-A_{3}$ & $A_{1}+A_{3}, B_{1}, B_{3}$ \\
$e_{3,4}$ & $B_{3}, A_{2}, A_{1}-A_{3}$ & $A_{1}+A_{3}, B_{1}, B_{2}$ \\
$e_{3,3} ; e_{3,5} ; e_{3,6} ; e_{3,7}$ & $A_{2}+\alpha B_{2}, A_{1}-A_{3}, B_{1}-B_{3}$ & $A_{1}+A_{3}, B_{1}+B_{3}, B_{2}$ \\
\hline
\end{tabular}

Equations (A.1) and (A.3) give $\tilde{\omega}^{1}=\tilde{\omega}^{2}=\tilde{\omega}^{3}=0$. Equation (A.5) has the general solution $\left\{r_{k}^{\alpha}=0\right\}_{\alpha=1 \ldots 3, k=1 \ldots 4}$, and $\omega^{\alpha} \otimes \mathbf{e}_{\alpha}$ defines a $G$-invariant connection.

Subcase 1.4: The following 2-dimensional algebras in [16] always admit a solution to (A.5). Therefore, they lead to the construction of a reductive homogeneous space.

\begin{tabular}{|l|c|c|}
\hline $\mathbf{h}$ & Basis $\left\{\mathbf{b}_{1}, \mathbf{b}_{2}\right\}$ & Complement $\left\{\tilde{\mathbf{b}}_{1}, \tilde{\mathbf{b}}_{2}, \tilde{\mathbf{b}}_{3}, \tilde{\mathbf{b}}_{4}\right\}$ \\
\hline$e_{2,3}$ & $B_{2}, A_{1}-A_{3}$ & $A_{1}+A_{3}, A_{2}, B_{1}, B_{3}$ \\
$e_{2,4}$ & $A_{1}-A_{3}, B_{3}$ & $A_{1}+A_{3}, A_{2}, B_{1}, B_{2}$ \\
$e_{2,7}$ & $A_{2}, A_{1}-A_{3}$ & $A_{1}+A_{3}, B_{1}, B_{2}, B_{3}$ \\
$e_{2,10} ; e_{2,11}$ & $A_{2}+c B_{2},-A_{1}+A_{3} ; c>0$ & $A_{1}+A_{3}, B_{1}, B_{2}, B_{3}$ \\
$e_{2,12}$ & $A_{2}-c B_{3},-A_{1}+A_{3} ; c \neq 0$ & $A_{1}+A_{3}, B_{1}, B_{2}, B_{3}$ \\
$e_{2,13}^{\epsilon}$ & $B_{2}+\epsilon\left(A_{3}-A_{1}\right), B_{1}-B_{3} ; \epsilon= \pm 1$ & $A_{1}+A_{3}, A_{2}, B_{1}+B_{3}, B_{2}$ \\
\hline
\end{tabular}

Equations (A.1) and (A.3) give $\tilde{\omega}^{1}=\tilde{\omega}^{2}=\tilde{\omega}^{3}=\tilde{\omega}^{4}=0$. Equation (A.5) has the general solution $\left\{r_{k}^{\alpha}=0\right\}_{\alpha=1 \ldots 2, k=1 \ldots 4}$, and $\omega^{\alpha} \otimes \mathbf{e}_{\alpha}$ defines a $G$-invariant connection.

Subcase 1.4.a: The subalgebras $e_{2,8}$ and $e_{2,9}$ in [16] are $\mathbf{h}=\operatorname{span}\left\{\mathbf{b}_{1}=A_{2}+B_{2}, \mathbf{b}_{2}=-A_{1}+\right.$ $\left.A_{3}+\epsilon\left(B_{1}-B_{3}\right)\right\}$ when $\epsilon=1$ and $\epsilon=-1$ respectively. By using the basis $\left\{\tilde{\mathbf{b}}_{1}=A_{1}+A_{3}, \tilde{\mathbf{b}}_{2}=\right.$ $\left.B_{1}+B_{3}, \tilde{\mathbf{b}}_{3}=A_{2}-B_{3}, \tilde{\mathbf{b}}_{3}=-A_{1}+A_{3}-\epsilon\left(B_{1}-B_{3}\right), \mathbf{b}_{1}, \mathbf{b}_{2}\right\}$ for $\mathbf{o}(2,2)$, equations (A.1) and (A.3) give

$$
\tilde{\omega}^{1}=\epsilon\left(2 p_{2}-p_{1}\right) \theta^{1}, \quad \tilde{\omega}^{2}=p_{1} \theta^{1}, \quad \tilde{\omega}^{3}=p_{2}\left(\theta^{4}-\epsilon \theta^{3}\right), \quad \tilde{\omega}^{4}=p_{2} \theta^{2} .
$$

Equation (A.5) has the general solution

$$
r_{1}^{1}=r_{2}^{1}=r_{1}^{2}=r_{3}^{2}=r_{4}^{2}=0, \quad r_{3}^{1}=\epsilon\left(p_{2}-p_{1}\right), \quad r_{4}^{1}=r_{2}^{2}=p_{1}-p_{2},
$$

and $\left(\omega^{1}+\left(p_{1}-p_{2}\right)\left(\theta^{4}-\epsilon \theta^{3}\right)\right) \otimes \mathbf{e}_{1}+\left(\omega^{2}+\left(p_{1}-p_{2}\right) \theta^{2}\right) \otimes \mathbf{e}_{2}$ defines a $G$-invariant connection.

We now consider the cases where condition (A.5) is not automatically satisfied.

Case 2: The subalgebra $e_{2,1}$ in [16] is $\mathbf{h}=\operatorname{span}\left\{A_{1}-A_{3}, B_{1}-B_{3}\right\}$. By using the basis $\left\{\tilde{\mathbf{b}}_{1}=\right.$ $\left.A_{1}+A_{3}, \tilde{\mathbf{b}}_{2}=A_{2}, \tilde{\mathbf{b}}_{3}=B_{1}+B_{3}, \tilde{\mathbf{b}}_{4}=B_{2}, \mathbf{b}_{1}, \mathbf{b}_{2}\right\}$ for $\mathbf{o}(2,2)$, equations (A.1) and (A.3) give

$$
\tilde{\omega}^{1}=p_{1} \theta^{1}, \quad \tilde{\omega}^{2}=p_{2} \theta^{1}-2 p_{1} \theta^{3}, \tilde{\omega}^{3}=p_{3} \theta^{1}, \quad \tilde{\omega}^{4}=p_{4} \theta^{1}-2 p_{3} \theta^{4} .
$$


From condition (A.5), $G / H$ is reductive if and only if $p_{1}=0$ and $p_{3}=0$. The first Bianchi identity yields

$$
\begin{array}{llll}
C_{12}^{1}=\frac{1}{2}\left(p_{2}+3 p_{4}\right) p_{3}, & C_{34}^{1}=\frac{3}{2}\left(p_{4}-p_{2}\right) p_{3}, & C_{24}^{2}=-2 p_{1} p_{3}, & C_{23}^{2}=0, \\
C_{12}^{2}=\frac{1}{2}\left(p_{4}+3 p_{2}\right) p_{1}, & C_{34}^{2}=\frac{3}{2}\left(p_{4}-p_{2}\right) p_{1}, & C_{23}^{2}=-2 p_{1} p_{3}, & C_{24}^{1}=0 .
\end{array}
$$

The second Bianchi identity has the general solution

$$
p_{2}=0, \quad p_{4}=0, \quad C_{13}^{2}=t p_{1}^{2}, \quad C_{14}^{1}=t p_{3}^{2}, \quad C_{14}^{2}=\frac{5}{3} t p_{1} p_{3}
$$

where $t \in \mathbb{R}$. Let $L=2 p_{1} p_{3}$. The curvature components are

$$
\begin{aligned}
& \Omega_{12}=L \theta^{1} \wedge \theta^{2}, \quad \Omega_{23}=-L \theta^{1} \wedge \theta^{4}, \quad \Omega_{13}=t p_{1}^{2} \theta^{1} \wedge \theta^{3}+\frac{5}{6} t L \theta^{1} \wedge \theta^{4}-L \theta^{2} \wedge \theta^{4}, \\
& \Omega_{34}=L \theta^{3} \wedge \theta^{4}, \quad \Omega_{24}=-L \theta^{1} \wedge \theta^{3}, \quad \Omega_{14}=\frac{5}{6} t L \theta^{1} \wedge \theta^{3}+t p_{3}{ }^{2} \theta^{1} \wedge \theta^{4}-L \theta^{2} \wedge \theta^{3} .
\end{aligned}
$$

The Jacobi identities are now satisfied, but depending on the parameters we get non-isomorphic Lie algebras. We now determine the non-isomorphic algebras.

Subcase 2.1: If $p_{1} \neq 0$ and $p_{3} \neq 0$ the change of basis

$$
\begin{array}{lll}
\alpha^{1}=-p_{1} \theta^{3}-p_{3} \theta^{4}, & \alpha^{3}=L\left(\frac{1}{3} t \theta^{1}-\theta^{2}\right)-p_{1} \omega^{1}-p_{3} \omega^{2}, & \alpha^{4}=\sqrt{2}\left(p_{1} \theta^{3}-p_{3} \theta^{4}\right), \\
\alpha^{2}=-\theta^{1}, & \alpha^{6}=-L\left(\frac{2}{3} t \theta^{1}+\theta^{2}\right)+p_{1} \omega^{1}+p_{3} \omega^{2}, & \alpha^{5}=\sqrt{2}\left(p_{1} \omega^{5}-p_{3} \omega^{2}\right)
\end{array}
$$

for $\mathbf{g}^{*}$ leads to the multiplication table $\mathbf{B 2}$ in Theorem 2.3 with isotropy $\mathbf{h}=\operatorname{span}\left\{\mathbf{e}_{5}, \mathbf{e}_{3}-\mathbf{e}_{6}\right\}$.

Subcase 2.2: If $p_{1}=0$ or $p_{3}=0$ the change of basis

$$
\begin{array}{lll}
\alpha^{1}=-p_{1} \theta^{3}-p_{3} \theta^{4}, & \alpha^{3}=p_{1} \omega^{1}+p_{3} \omega^{2}, & \alpha^{5}=\left(p_{3}{ }^{2}-p_{1}{ }^{2}\right)\left(\frac{1}{3} t \theta^{1}-\theta^{2}\right)-p_{3} \omega^{1}+p_{1} \omega^{2}, \\
\alpha^{2}=\theta^{1}, & \alpha^{4}=p_{3} \theta^{3}-p_{1} \theta^{4}, & \alpha^{6}=\frac{1}{2} t\left(p_{3}+p_{1}\right)^{2} \theta^{2}-\left(p_{3}{ }^{3} \omega^{1}+p_{1}{ }^{3} \omega^{2}\right) /\left(p_{3}+p_{1}\right)^{2},
\end{array}
$$

for $\mathbf{g}^{*}$ leads to the multiplication table B3 in Theorem 2.3 with isotropy in the dual basis $\mathbf{h}=$ $\operatorname{span}\left\{\mathbf{e}_{3}, \mathbf{e}_{5}+\mathbf{e}_{6}\right\}$ when $p_{1}=0$ and $\mathbf{h}=\operatorname{span}\left\{\mathbf{e}_{3}, \mathbf{e}_{5}-\mathbf{e}_{6}\right\}$ when $p_{3}=0$. Reversing the sign of $\mathbf{e}_{6}$ is an automorphism, thus these are equivalent Lie algebra pairs.

Case 3: The subalgebra $e_{1,10}$ in $[16]$ is $\mathbf{h}=\operatorname{span}\left\{-A_{1}+A_{3}\right\}$. By using the basis $\left\{\tilde{\mathbf{b}}_{1}=A_{1}+A_{3}, \tilde{\mathbf{b}}_{2}=\right.$ $\left.A_{2}, \tilde{\mathbf{b}}_{3}=B_{1}, \tilde{\mathbf{b}}_{4}=B_{2}, \tilde{\mathbf{b}}_{5}=B_{3}, \mathbf{b}_{1}=-A_{1}+A_{3}\right\}$ for $\mathbf{o}(2,2)$, equations (A.1) and (A.3) give

$$
\begin{array}{ll}
\tilde{\omega}^{1}=p_{1} \theta^{1}+p_{2} \theta^{4}, \quad \tilde{\omega}^{3}=\left(p_{5}+p_{9}\right) \theta^{1}+\left(p_{6}-p_{3}\right) \theta^{4}, & \tilde{\omega}^{4}=\left(p_{3}+p_{7}\right) \theta^{1}+p_{8} \theta^{4}, \\
\tilde{\omega}^{2}=\frac{1}{3}\left(p_{3}-p_{7}\right) \theta^{1}+2 p_{2} \theta^{2}-2 p_{1} \theta^{3}+\frac{1}{3}\left(p_{4}-p_{9}\right) \theta^{4}, & \tilde{\omega}^{5}=\left(p_{9}-p_{5}\right) \theta^{1}+p_{10} \theta^{4} .
\end{array}
$$

From condition (A.5), $G / H$ is reductive if and only if $p_{1}=0$ and $p_{2}=0$. The first Bianchi identity yields

$$
\begin{aligned}
& p_{3}=s p_{1}, \quad p_{4}=t p_{1}, \quad p_{5}=-r p_{1}, \quad p_{8}=t p_{1}+s p_{2}, \quad C_{12}^{1}=5 p_{1} p_{2} J, \quad C_{13}^{1}=-5 p_{1}^{2} J, \quad C_{23}^{1}=-K, \\
& p_{9}=s p_{2}, \quad p_{6}=t p_{2}, \quad p_{7}=r p_{2}, \quad p_{10}=t p_{2}+s p_{1}, \quad C_{34}^{1}=5 p_{1} p_{2} J, \quad C_{24}^{1}=-5 p_{2}^{2} J
\end{aligned}
$$

where $r, s, t \in \mathbb{R}$ and

$$
J=\frac{1}{3}\left(r t-s^{2}\right), \quad K=\frac{1}{3}\left(p_{1}^{2} t-2 p_{1} p_{2} s+p_{2}^{2} r\right), \quad L=\frac{1}{3}\left(p_{1}{ }^{2} t+4 p_{1} p_{2} s+p_{2}{ }^{2} r\right) .
$$


The second Bianchi identity has the general solution

$$
C_{14}^{1}=4 J K .
$$

The curvature components are

$$
\begin{aligned}
& \Omega_{12}=p_{1}\left(s p_{1}+r p_{2}\right) \theta^{1} \wedge \theta^{3}-(K+L) \theta^{1} \wedge \theta^{2}-5 p_{1} p_{2} J \theta^{1} \wedge \theta^{4}+p_{2}\left(t p_{1}+s p_{2}\right) \theta^{2} \wedge \theta^{4}-L \theta^{3} \wedge \theta^{4}, \\
& \Omega_{34}=p_{1}\left(s p_{1}+r p_{2}\right) \theta^{1} \wedge \theta^{3}-(K+L) \theta^{3} \wedge \theta^{4}-5 p_{1} p_{2} J \theta^{1} \wedge \theta^{4}+p_{2}\left(t p_{1}+s p_{2}\right) \theta^{2} \wedge \theta^{4}-L \theta^{1} \wedge \theta^{2}, \\
& \Omega_{13}=p_{1}\left(s p_{1}+r p_{2}\right) \theta^{1} \wedge \theta^{2}-2 r p_{1}^{2} \theta^{1} \wedge \theta^{3}+5 p_{1}^{2} J \theta^{1} \wedge \theta^{4}-2 s p_{1} p_{2} \theta^{2} \wedge \theta^{4}+p_{1}\left(s p_{1}+r p_{2}\right) \theta^{3} \wedge \theta^{4}, \\
& \Omega_{24}=p_{2}\left(t p_{1}+s p_{2}\right) \theta^{1} \wedge \theta^{2}-2 t p_{2}^{2} \theta^{2} \wedge \theta^{4}+5 p_{2}{ }^{2} J \theta^{1} \wedge \theta^{4}-2 s p_{1} p_{2} \theta^{1} \wedge \theta^{3}+p_{2}\left(t p_{1}+s p_{2}\right) \theta^{3} \wedge \theta^{4}, \\
& \Omega_{14}=J\left(5 p_{1} p_{2} \theta^{2}-5 p_{1}^{2} \theta^{3}+4 K \theta^{4}\right) \wedge \theta^{1}+K \theta^{2} \wedge \theta^{3}+5 J\left(p_{2}^{2} \theta^{2}-p_{1} p_{2} \theta^{3}\right) \wedge \theta^{4}, \\
& \Omega_{23}=K \theta^{1} \wedge \theta^{4} .
\end{aligned}
$$

The change of basis

$$
\begin{aligned}
& \alpha^{1}=\frac{1}{3}\left(2 s p_{1}-r p_{2}\right) \theta^{1}+p_{2} \theta^{2}-p_{1} \theta^{3}+\frac{1}{3} t p_{1} \theta^{4}, \quad \alpha^{2}=-p_{1} \theta^{1}-p_{2} \theta^{4} \\
& \alpha^{5}=\frac{1}{3}\left(2 s p_{2}+r p_{1}\right) \theta^{1}-p_{1} \theta^{2}-p_{2} \theta^{3}+\frac{1}{3} t p_{2} \theta^{4}, \quad \alpha^{4}=p_{2} \theta^{1}-p_{1} \theta^{4} \\
& \alpha^{3}=\frac{1}{9} r\left(3 t p_{1}-2 s p_{2}\right) \theta^{1}+\frac{1}{3}\left(t p_{1}+s p_{2}\right) \theta^{2}+\frac{1}{3}\left(r p_{2}-3 s p_{1}\right) \theta^{3}+\frac{1}{9}\left(2 s t p_{1}+3 t r p_{2}-4 s^{2} p_{2}\right) \theta^{4}+\omega^{1}
\end{aligned}
$$

for $\mathbf{g}^{*}$ leads to the multiplication table $\mathbf{B} \mathbf{1}$ in Theorem 2.3, with isotropy $\mathbf{h}=\operatorname{span}\left\{\mathbf{e}_{3}\right\}$.

Case 4: The subalgebras $e_{1,3}$ and $e_{1,4}$ in [16] satisfy Lemma 3.3. Define $A \in G L(4, \mathbb{R})$ by $A\left(f_{1}\right)=$ $f_{3}, A\left(f_{2}\right)=-f_{4}, A\left(f_{3}\right)=f_{1}, A\left(f_{4}\right)=-f_{2}$, where $f_{k}$ is the standard basis for $\mathbb{R}^{4}$, then $A$ is an automorphism of $\mathbf{o}(2,2)$ that maps $e_{1,3}$, which has basis $\left\{\mathbf{b}_{1}=-A_{1}+A_{3}-B_{1}+B_{3}\right\}$, to the subalgebra $e_{1,4}$, which has basis $\left\{\mathbf{b}_{1}=-A_{1}+A_{3}+B_{1}-B_{3}\right\}$. Therefore, we consider only $e_{1,4}$. By using the basis $\left\{\tilde{\mathbf{b}}_{1}=A_{1}-A_{3}+B_{1}-B_{3}, \tilde{\mathbf{b}}_{2}=B_{2}, \tilde{\mathbf{b}}_{3}=A_{1}-A_{3}+B_{1}+B_{3}, \tilde{\mathbf{b}}_{4}=-A_{1}-A_{3}+B_{1}-B_{3}, \tilde{\mathbf{b}}_{5}=A_{2}, \mathbf{b}_{1}\right\}$ for $\mathbf{o}(2,2)$, equations (A.1) and (A.3) give

$$
\begin{array}{ll}
\tilde{\omega}^{1}=p_{1} \theta^{1}+p_{2} \theta^{2}+\left(p_{3}+p_{4}\right) \theta^{3}+\left(p_{4}-p_{3}\right) \theta^{4}, & \tilde{\omega}^{3}=\left(p_{7}-p_{2}\right) \theta^{1}, \\
\tilde{\omega}^{2}=2\left(p_{5}-p_{3}\right) \theta^{1}+\left(p_{6}+p_{7}-p_{2}\right) \theta^{3}+\left(p_{6}-p_{7}+p_{2}\right) \theta^{4}, & \tilde{\omega}^{4}=\left(p_{7}+p_{2}\right) \theta^{1}, \\
\tilde{\omega}^{5}=2\left(p_{5}+p_{3}\right) \theta^{1}+\left(p_{6}+p_{7}+p_{2}\right) \theta^{3}+\left(p_{6}-p_{7}-p_{2}\right) \theta^{4} . &
\end{array}
$$

From condition (A.5), $G / H$ is reductive if and only if $p_{6}=0$. The first Bianchi identity yields

$$
\begin{array}{lll}
p_{4}=t\left(p_{6}+p_{2}\right), & C_{24}^{1}=p_{7}^{2}, & C_{34}^{1}=2 p_{3} p_{7}+2 p_{2} p_{4}, \quad C_{12}^{1}=4 p_{2} p_{3}-4 p_{3} p_{6}-4 p_{5} p_{7}, \\
p_{5}=t\left(p_{2}-p_{6}\right), & C_{23}^{1}=-p_{7}^{2}, & C_{14}^{1}=-4 p_{3} p_{4}+2 p_{2}^{2}-6 p_{1} p_{7}-6 p_{7}^{2}+4 p_{2} p_{6}+4 p_{5} p_{3}-C_{13}^{1}
\end{array}
$$

where $t \in \mathbb{R}$, and the conditions

$$
p_{2} p_{7}=0, \quad 2 p_{2} p_{3}+\left(p_{3}-t p_{7}\right) p_{6}=0 .
$$

Let $K=\left(2 p_{6} t^{2}+p_{1}+p_{7}\right) / p_{6}, L=2\left(p_{6} t^{2}+p_{7}+p_{1}\right) / p_{6}$ and $\beta=C_{13}^{1}-p_{2}^{2}-2 p_{2} p_{6}-2 p_{2} p_{6} t^{2}$. The second Bianchi identity yields $p_{3}=t p_{7}$ so the final the remaining conditions are

$$
p_{2} p_{7}=0, \quad \beta\left(p_{6}+p_{2}\right)+p_{7} p_{6}\left(L\left(p_{6}+2 p_{7}\right)+K p_{6}\right)=0 .
$$


This case splits into three subcases based on $p_{7}$ and $\beta$.

Subcase 4.1: If $p_{7} \neq 0$ then $p_{2}=0$. The remaining condition implies $C_{13}^{1}=-p_{7}\left(L\left(p_{6}+2 p_{7}\right)+K p_{6}\right)$. The curvature components are

$$
\begin{aligned}
\Omega_{23}= & p_{7}^{2} \theta^{1} \wedge\left(\theta^{4}-\theta^{3}\right), \quad \Omega_{12}=-2 p_{7}^{2} \theta^{1} \wedge\left(\theta^{2}+t \theta^{3}+t \theta^{4}\right), \\
\Omega_{24}= & p_{7}^{2} \theta^{1} \wedge\left(\theta^{3}-\theta^{4}\right), \quad \Omega_{34}=2 t p_{7}^{2} \theta^{1} \wedge\left(\theta^{3}-\theta^{4}\right), \\
\Omega_{13}= & -2 t p_{7}^{2} \theta^{1} \wedge \theta^{2}-2\left(p_{6}+p_{7}\right)\left(p_{6}+2 p_{7}\right) K \theta^{1} \wedge \theta^{3}-2\left(p_{6}{ }^{2} K-p_{7}^{2} L\right) \theta^{1} \wedge \theta^{4} \\
& -p_{7}^{2} \theta^{2} \wedge\left(\theta^{3}-\theta^{4}\right)+2 t p_{7}^{2} \theta^{3} \wedge \theta^{4}, \\
\Omega_{14}= & -2 t p_{7}^{2} \theta^{1} \wedge \theta^{2}-2\left(p_{6}-p_{7}\right)\left(p_{6}-2 p_{7}\right) K \theta^{1} \wedge \theta^{4}-2\left(p_{6}{ }^{2} K-p_{7}^{2} L\right) \theta^{1} \wedge \theta^{3} \\
& +p_{7}^{2} \theta^{2} \wedge\left(\theta^{3}-\theta^{4}\right)-2 t p_{7}^{2} \theta^{3} \wedge \theta^{4},
\end{aligned}
$$

The change of basis

$$
\begin{array}{ll}
\alpha^{1}=-2 p_{6} t \theta^{1}+\left(p_{6}+2 p_{7}\right) \theta^{3}+\left(p_{6}-2 p_{7}\right) \theta^{4}, & \alpha^{2}=-4 p_{7}^{2} / p_{6} \theta^{1}, \\
\alpha^{3}=\left(p_{1}+p_{7}\right) \theta^{1}+p_{6} \theta^{2}+p_{6} t \theta^{3}+p_{6} t \theta^{4}-p_{6} / p_{7} \omega^{1}, & \alpha^{5}=-2 p_{6} t \theta^{1}+p_{6} \theta^{3}+p_{6} \theta^{4}, \\
\alpha^{4}=2\left(p_{1}+p_{7}\right) \theta^{1}-p_{6} \theta^{2}+p_{6} t \theta^{3}+p_{6} t \theta^{4}-p_{6} / p_{7} \omega^{1} &
\end{array}
$$

for $\mathbf{g}^{*}$ leads to the multiplication table $\mathbf{A} \mathbf{1}$ in Theorem 2.3 with isotropy in the dual basis $\mathbf{h}=$ $\operatorname{span}\left\{\mathbf{e}_{3}+\mathbf{e}_{4}\right\}$.

Subcase 4.2: If $p_{7}=0$ the remaining the condition splits into two subcases based on $\beta$.

Subcase 4.2.a: When $\beta=0$, the remaining condition implies $C_{13}^{1}=p_{2}{ }^{2}+2 p_{2} p_{6}+2 p_{2} p_{6} t^{2}$. The curvature components are

$$
\begin{aligned}
& \Omega_{12}=2 p_{2}^{2} \theta^{1} \wedge \theta^{2}, \quad \Omega_{23}=-2 p_{2}^{2} \theta^{1} \wedge \theta^{4}, \quad \Omega_{24}=-2 p_{2}^{2} \theta^{1} \wedge \theta^{3}, \quad \Omega_{34}=2 p_{2}{ }^{2} \theta^{3} \wedge \theta^{4}, \\
& \Omega_{13}=-K \theta^{1} \wedge\left(\left(p_{2}+2 p_{6}\right) \theta^{3}+\left(3 p_{2}+2 p_{6}\right) \theta^{4}\right)-2 p_{2}{ }^{2} \theta^{2} \wedge \theta^{4}, \\
& \Omega_{14}=-K \theta^{1} \wedge\left(\left(p_{2}+2 p_{6}\right) \theta^{4}+\left(3 p_{2}+2 p_{6}\right) \theta^{3}\right)-2 p_{2}{ }^{2} \theta^{2} \wedge \theta^{3} .
\end{aligned}
$$

The change of basis

$$
\begin{aligned}
& \alpha^{1}=-p_{1} \theta^{1}+2 p_{6} \theta^{2}, \quad \alpha^{2}=2 p_{6}\left(\theta^{3}-\theta^{4}\right), \quad \alpha^{3}=4 p_{6} \theta^{1}, \quad \alpha^{4}=p_{2}\left(\theta^{1}+t \theta^{3}-t \theta^{4}\right)+\omega^{1}, \\
& \alpha^{5}=p_{6}\left(-2 t \theta^{1}+\theta^{3}+\theta^{4}\right)
\end{aligned}
$$

for $\mathbf{g}^{*}$ leads to the multiplication table $\mathbf{A 2}$ in Theorem 2.3 where $\alpha=-p_{2} / p_{6}$ with isotropy in the dual basis $\mathbf{h}=\operatorname{span}\left\{\mathbf{e}_{4}\right\}$.

Subcase 4.2.b: If $\beta \neq 0$ then $p_{2}=-p_{6}$. The curvature components are

$$
\begin{array}{lll}
\Omega_{12}=2 p_{6}{ }^{2} \theta^{1} \wedge \theta^{2}, & \Omega_{23}=-2 p_{6}{ }^{2} \theta^{1} \wedge \theta^{4}, & \Omega_{13}=\left(\beta-p_{6}{ }^{2} K\right) \theta^{1} \wedge\left(\theta^{3}-\theta^{4}\right)-2 p_{6}{ }^{2} \theta^{2} \wedge \theta^{4}, \\
\Omega_{34}=2 p_{6}{ }^{2} \theta^{3} \wedge \theta^{4}, & \Omega_{24}=-2 p_{6}{ }^{2} \theta^{1} \wedge \theta^{3}, & \Omega_{14}=\left(\beta-p_{6}{ }^{2} K\right) \theta^{1} \wedge\left(\theta^{4}-\theta^{3}\right)-2 p_{6}{ }^{2} \theta^{2} \wedge \theta^{3} .
\end{array}
$$

The change of basis

$$
\begin{array}{ll}
\alpha^{1}=\sqrt{|\beta / 2|}\left(\frac{1}{2} p_{1} / p_{6} \theta^{1}-\theta^{2}\right), & \alpha^{3}=p_{6}\left(\theta^{1}+t \theta^{3}-t \theta^{4}\right)-\omega^{1}, \quad \alpha^{5}=\sqrt{|2 \beta|} \theta^{1}, \\
\alpha^{2}=\sqrt{|\beta / 2|}\left(\theta^{3}-\theta^{4}\right), & \alpha^{4}=p_{6}\left(-2 t \theta^{1}+\theta^{3}+\theta^{4}\right),
\end{array}
$$

for $\mathbf{g}^{*}$ leads to the multiplication table $\mathbf{A} \mathbf{3}$ in Theorem 2.3 where $\epsilon=\frac{\beta}{|\beta|}$ and the isotropy in the dual basis is $\mathbf{h}=\operatorname{span}\left\{\mathbf{e}_{3}\right\}$.

This concludes the proof of Theorem 2.3.

We now list the algebra pairs in Theorems 2.2 and 2.3 where the metrics can be Einstein without being of constant curvature. 
Lemma 5.1. Let $(G / H, \eta)$ be a homogeneous non-reductive pseudo-Riemannian Einstein manifold of dimension four which is not of constant curvature and where $H$ is connected.

i] The space is Ricci-flat if and only if the Lie algebra pair $(\mathbf{g}, \mathbf{h})$ is isomorphic to $\mathbf{B 3}$.

ii] The space is Einstein and not Ricci flat if and only if the Lie algebra is isomorphic to the pair A2 with $\alpha=2 / 3$.

Proof. Starting with the curvature forms in equations (5.2), (5.5), (5.7), (5.9), (5.11), (5.13), (5.15), (5.17), (5.19), the coefficients of the Ricci tensor in an orthonormal frame $u_{\sigma}$ at $\sigma=[H]$ are easily computed. For example, from the curvature in (5.7) we get

$$
\text { Ricci }=\left(3 \alpha^{2} p_{4}^{2}\right) \eta^{0}-p_{4}\left(t_{1}^{2} p_{4}+p_{7}\right)(3 \alpha-2) \theta^{3} \otimes \theta^{3}
$$

where $p_{4} \neq 0$. If $\alpha=\frac{2}{3}$ and $t_{1}^{2} p_{4}+p_{4} \neq 0$ the space is Einstein and not of constant curvature. Similar computations with (5.17) and (5.11) in Subcase 2.2 prove the lemma.

Case i] in Lemma 5.1 corresponds to Proposition 2.5.2 on pg. 153 of [10], and case ii] in Lemma 5.1 corresponds to Proposition 1.4.2 on pg. 142 of [10].

\section{Global Results and Existence}

To prove Theorem 2.4, we start by characterizing the four-dimensional simply connected nonreductive pseudo-Riemannian homogeneous spaces. These turn out to be fairly simple.

Theorem 6.1. Let $(\mathbf{g}, \mathbf{h})$ be a Lie algebra pair from Theorem 2.2 or Theorem 2.3 and suppose $G$ is the simply connected Lie group with Lie algebra $\mathbf{g}$. Then there exists a closed connected Lie subgroup $H \subset G$ with Lie algebra $\mathbf{h}$ such that $G / H$ is diffeomorphic to $\mathbb{R}^{4}$.

Proof. The proof is done on a case by case basis. We first consider the pairs in A2-A3 of Theorem 2.2 (or Theorem 2.3) where $\mathbf{g}$ is solvable. Let $G$ be the simply connected solvable Lie group having Lie algebra $\mathbf{g}$, and $H$ be the connected Lie subgroup having Lie subalgebra $\mathbf{h}$. Since $H$ is closed [4], $G / H$ is a manifold. Since $H$ is connected, $G / H$ is simply connected and $G / H$ is diffeomorphic to $\mathbb{R}^{4}[13]$.

For cases A1 and A4 of Theorem 2.2 and B1-B3 of Theorem 2.3, we construct connected Lie groups $G^{0}$ and connected closed subgroups $H^{0} \subset G^{0}$ such that the covering space of $G^{0} / H^{0}$ is $\mathbb{R}^{4}$. It follows that $\mathbb{R}^{4}=\tilde{G} / H$ (see Theorem 2.1 p. $125[14]$ ) where $\tilde{G}$ is the simply connected cover of $G^{0}$ and $H$ is a closed connected Lie subgroup having Lie subalgebra $\mathbf{h}$. We start with $\mathbf{A 4}$ and B2.

Let $\mathbf{a}, \mathbf{b} \in \mathbb{R}^{2}$, and $\mathbf{a} \times \mathbf{b}=a_{1} b_{2}-b_{1} a_{2}$. The multiplication map for the six-dimensional Lie group $S L(2, \mathbb{R}) \ltimes N_{3}$ is

$$
(A, \mathbf{a}, \alpha) *(B, \mathbf{b}, \beta)=\left(A A^{\prime}, A \mathbf{b}+\mathbf{a}, \alpha+\beta-(A \mathbf{b}) \times \mathbf{a}\right)
$$

where $A, B \in S L(2, \mathbb{R}), \mathbf{a}, \mathbf{b} \in \mathbb{R}^{2}$ and $\alpha, \beta \in \mathbb{R}$. Let $H_{l}^{0}$ and $H_{n}^{0}$ be the closed subgroups

$$
\left.\left.H_{l}^{0}=\left\{\left(\begin{array}{ll}
1 & 0 \\
t & 1
\end{array}\right),\left(\begin{array}{l}
0 \\
s
\end{array}\right), 2 t\right) \quad t, s \in \mathbb{R}\right\}, \quad H_{n}^{0}=\left\{\left(\begin{array}{ll}
1 & 0 \\
t & 1
\end{array}\right),\left(\begin{array}{l}
0 \\
s
\end{array}\right),-2 t\right) \quad t, s \in \mathbb{R}\right\} .
$$

The Lie algebra pair in $\mathbf{A 4}$ is isomorphic to $\left(\mathbf{g}, \mathbf{h}_{l}\right)$, and the pair in $\mathbf{B 2}$ is isomorphic to $\left(\mathbf{g}, \mathbf{h}_{n}\right)$. The quotient spaces $G^{0} / H_{l}^{0}$ and $G^{0} / H_{n}^{0}$ are diffeomorphic to $\left(\mathbb{R}^{2} \backslash\{(0,0)\}\right) \times \mathbb{R}^{2}$, so the covering space in these cases is $\mathbb{R}^{4}$.

For the Lie algebra pair B1 in Theorem 2.3, let $G^{0}$ be the group $S L(2, \mathbb{R}) \ltimes \mathbb{R}^{2}$, and let

$$
H^{0}=\left\{\left(\begin{array}{ll}
1 & 0 \\
t & 1
\end{array}\right),\left(\begin{array}{l}
0 \\
0
\end{array}\right) \quad t \in \mathbb{R}\right\}
$$


The pair in B1 of Theorem 2.3 is isomorphic to this $(\mathbf{g}, \mathbf{h})$. The quotient space $G^{0} / H^{0}$ is diffeomorphic to $\left(\mathbb{R}^{2} \backslash\{(0,0)\}\right) \times \mathbb{R}^{2}$, so its simply connected cover is $\mathbb{R}^{4}$. The Lie algebra pair in $\mathbf{A} \mathbf{1}$ in Theorems 2.2 and 2.3 is similar to this one.

For $\mathbf{A 5}{ }^{*}$, consider the monomorphism $\phi: \mathbf{g} \rightarrow \mathbf{o}(2,3)$ given by

$$
\begin{aligned}
& \phi\left(\mathbf{e}_{1}\right)=e_{4}^{1}+e_{1}^{4}-e_{3}^{2}-e_{2}^{3}, \quad \phi\left(2 \mathbf{e}_{2}\right)=e_{2}^{1}+e_{3}^{1}+e_{4}^{2}+e_{1}^{3}+e_{2}^{4}+e_{3}^{4}-e_{1}^{2}-e_{4}^{3}, \\
& \phi\left(\sqrt{2} \mathbf{e}_{5}\right)=e_{5}^{1}+e_{1}^{5}+e_{4}^{5}-e_{5}^{4}, \phi\left(2 \mathbf{e}_{3}\right)=e_{3}^{1}+e_{1}^{2}+e_{4}^{2}+e_{1}^{3}+e_{4}^{3}+e_{2}^{4}-e_{2}^{1}-e_{3}^{4}, \\
& \phi\left(\sqrt{2} \mathbf{e}_{6}\right)=e_{5}^{3}-e_{5}^{2}-e_{2}^{5}-e_{3}^{5}, \quad \phi\left(2 \mathbf{e}_{4}\right)=e_{1}^{2}+e_{4}^{2}+e_{2}^{4}+e_{3}^{4}-e_{2}^{1}-e_{3}^{1}-e_{1}^{3}-e_{4}^{3}, \\
& \phi\left(\mathbf{e}_{7}\right)=e_{4}^{1}+e_{3}^{2}+e_{2}^{3}+e_{1}^{4},
\end{aligned}
$$

where $\eta^{0}=\operatorname{diag}(-1,-1,1,1,1)$ is the symmetric $5 \times 5$ matrix defining $\mathbf{o}(2,3)$. Let $G$ be the simply connected seven-dimensional Lie group having Lie algebra $\mathbf{g}$, and let $\Phi: G \rightarrow O(2,3)$ be the induced homomorphism from $\phi$. We now show that $G$ acts transitively on the manifold

$$
M=\left\{\left(x_{1}, x_{2}, x_{3}, x_{4}, x_{5}\right) \in \mathbb{R}^{5} \mid-x_{1}{ }^{2}-x_{2}{ }^{2}+x_{3}{ }^{2}+x_{4}{ }^{2}+x_{5}{ }^{2}=-r^{2}\right\}
$$

which is diffeomorphic to $S^{1} \times \mathbb{R}^{3}[19]$. Let $\left(x_{1}, x_{2}, x_{3}, x_{4}, x_{5}\right) \in M$. Application of the group element $e^{\phi\left(t \mathbf{e}_{3}-t \mathbf{e}_{2}\right)}$, where $t=\pi / 2$ if $x_{2}=0$ otherwise $\tan t=x_{1} / x_{2}$, maps this point to $\left(0, \tilde{x}_{2}, \tilde{x}_{3}, \tilde{x}_{4}, \tilde{x}_{5}\right)$, where $\tilde{x}_{2} \neq 0$. Similar use of the one parameter subgroups of $G$ map this point to $(0, r, 0,0,0)$. Hence, $G$ acts transitively on $M$. The Lie algebra of the Lie subgroup of $G$ which stabilizes $(0, r, 0,0,0)$ is $\mathbf{h}=\left\{\mathbf{e}_{1}+\mathbf{e}_{7}, \mathbf{e}_{3}-\mathbf{e}_{4}, \mathbf{e}_{5}\right\}$. Therefore, the covering space for $G / H$ is $\mathbb{R}^{4}$.

The details for $\mathbf{B} 3$ can be found in the proof of Theorem 2.5 in section 7 .

Theorem 6.1 can now be used to prove Theorem $2.4 \mathrm{i}]$.

Proof. Proof of Theorem 2.4 i]: Since the homogeneous space $G / H$ in the theorem is simply connected, we may assume $G$ is simply connected and $H$ is connected. By Theorem 2.2 or Theorem 2.3, the Lie algebra pair $(\mathbf{g}, \mathbf{h})$ is isomorphic to one of $\mathbf{A 1 - A 5 *}$ or B1-B3. The Lie algebra isomorphism lifts to a Lie group isomorphism to one of the simply connected groups used in the proof of Theorem 6.1. Therefore, $M$ is diffeomorphic to $\mathbb{R}^{4}$.

Up to this point, we have shown that a simply connected non-reductive homogeneous pseudoRiemannian manifold is diffeomorphic to $\mathbb{R}^{4}$ and the Lie algebra of its isometry group must be isomorphic to one in Theorems 2.2 or 2.3. We now show that $\mathbf{A} 5^{*}$ cannot occur.

Lemma 6.1. Let $(G / H, \eta)$ be a simply connected four-dimensional homogeneous Lorentz manifold with Lie algebra pair A5*. Then $G$ is a proper subgroup of the isometry group $\tilde{O}(2,3)$.

Proof. The computations in Theorem 6.1 show that there exists a transitive action of $G$ on $S^{1} \times \mathbb{R}^{3}$ with isotropy $K$ which has the same Lie algebra as $H$. We showed in the proof of Theorem 2.2 in section 5 Case 2, that the Lorentz metric, which is unique up to scaling, was of constant (negative) curvature, so the standard action of $O(2,3)$ on $S^{1} \times \mathbb{R}^{3}$ is by isometries for this metric. Therefore, an invariant Lorentz metric on $G / H$ (the covering space) will admit $\tilde{O}(2,3)$ acting by isometries, and the Lie algebra of the isometry group will not be $\mathbf{g}$.

Lemma 6.1 allows us to prove Theorem 2.4 ii] by eliminating $\mathbf{A 5 *}$.

Proof. Proof of Theorem 2.4 ii]: Starting with $G / H$ simply connected we may assume that $H$ is connected and so Theorem 2.2, or 2.3 imply that the Lie algebra pair is isomorphic to one in the lists in these two theorems. However if $G$ is the isometry group then by Lemma $6.1, \mathbf{A 5}^{*}$ cannot 
be the Lie algebra pair for the isometry group of simply connected four-dimensional homogeneous Lorentz manifold.

In order to finish the proof of Theorem 2.4 (the converse part) we need to show that we can build metrics on $\mathbb{R}^{4}$ having the isometry algebras in Theorems 2.2 (except $\mathbf{A 5} 5^{*}$ ) and 2.3. In order to do this we first give two lemmas.

Lemma 6.2. Let $G / H$ be a homogeneous space with pair $(\mathbf{g}, \mathbf{h})$, and $H$ connected. If the pair $(\mathbf{g}, \mathbf{h})$ satisfies Lemma 3.2, then

$$
\eta(X, Y)=\eta^{0}(\theta(X), \theta(Y)) \quad X, Y \in T_{p} G
$$

is basic for the projection $\mathbf{q}: G \rightarrow G / H$ and defines a pseudo-Riemannian metric on $G / H$ with curvature tensor $\Omega$.

Proof. The form $\theta$ is $\mathbb{R}^{n}$ valued, so $\eta$ defines a symmetric bilinear form on $T G$. From 1] in Lemma 3.1, the form $\eta$ is semi-basic for the projection $\mathbf{q}: G \rightarrow G / H$ and has the same signature as $\eta^{0}$. The Lie derivative of $\eta$ with respect to $X \in \mathbf{h}$,

$$
\mathcal{L}_{X} \eta^{0}(\theta, \theta)=\eta^{0}\left(\mathcal{L}_{X} \theta, \theta\right)+\eta\left(\theta, \mathcal{L}_{X} \theta\right)=\eta^{0}\left(\rho_{*}(X) \theta, \theta\right)+\eta^{0}\left(\theta, \rho_{*}(X) \theta\right)=0
$$

implies that $\eta$ is $H$-basic, because $H$ is connected. We can check that $\Omega$ is the curvature of $\eta$, by choosing a local cross section of $\mathbf{q}: G \rightarrow G / H$ and pulling back the structure equations by the cross section, or by reversing the arguments in section 3 which we now do. Let $u_{\sigma} \in O(M)$, and $\Psi$ be constructed as in (3.5). The pullback $\Psi^{*} \bar{\theta}$ of the canonical form $\bar{\theta}$ on the frame bundle, are $G$-invariant and provide a basis for the $\mathbf{q}: G \rightarrow G / H$ semi-basic forms. Therefore,

$$
\theta=A \Psi^{*} \bar{\theta} \quad \text { where } \quad A \in G L(n, \mathbb{R})
$$

Now, let $X, Y \in \mathbb{R}^{n}$ and choose $\tilde{X}, \tilde{Y} \in T_{e} G$ such that $\mathbf{q}_{*} \tilde{X}=u_{\sigma} X$, and $\mathbf{q}_{*} \tilde{Y}=u_{\sigma} Y$. By definition of $u_{\sigma}$ and $\eta$, we have

$$
\eta^{0}(X, Y)=\eta\left(u_{\sigma} X, u_{\sigma} Y\right)=\eta^{0}(\theta(\tilde{X}), \theta(\tilde{Y})) .
$$

Condition (6.1) gives

$$
\eta^{0}(X, Y)=\eta^{0}\left(A \bar{\theta}\left(\Psi_{*} X\right), A \bar{\theta}\left(\Psi_{*} Y\right)\right)=\eta^{0}\left(A u_{\sigma}^{-1} \pi_{*} \Psi_{*} X, A u_{\sigma}^{-1} \pi_{*} \Psi_{*} X\right) .
$$

The commutative diagram (3.6) gives

$$
\eta^{0}(X, Y)=\eta^{0}\left(A \mathbf{q}_{*}(\tilde{X}), A \mathbf{q}_{*}(\tilde{Y})\right)=\eta^{0}(A X, A Y)
$$

so $A \in O(p, \tilde{p})$. Finally, using the frame $v_{\sigma}=u_{\sigma} A$ to redefine $\Psi$, we get $\Psi^{*} \theta=\theta, \Psi^{*} \omega=\omega$, and $\Psi^{*} \Omega=\Omega$.

This lemma says that for any case we consider in section 5 , and no matter what value we choose for the parameters in the curvature form $\Omega$, we can construct a homogeneous pseudo-Riemannian manifold having the chosen value of the curvature form. In the next lemma, we give a sufficienct condition on the curvature for a given Lie algebra to be the Lie algebra of the isometry group. 
Lemma 6.3. Let $(G / H, \eta)$ be an n-dimensional pseudo-Riemannian homogeneous space with curvature form $\Omega$ and let $R_{i j k l}$ and $R_{i j k l ; m}$ be the corresponding components of the Riemann curvature tensor and its covariant derivative in the orthonormal frame $u_{\sigma}$ at $\sigma=[H]$. Let

$$
\begin{aligned}
S=\{E \in \mathbf{o}(p, \tilde{p}) \mid & R_{s j k l} E_{i}^{s}+R_{i s k l} E_{j}^{s}+R_{i j s l} E_{k}^{s}+R_{i j k s} E_{l}^{s}=0, \\
& \left.R_{s j k l ; m} E_{i}^{s}+R_{i s k l ; m} E_{j}^{s}+R_{i j s l ; m} E_{k}^{s}+R_{i j k s ; m} E_{l}^{s}+R_{i j k l ; s} E_{m}^{s}=0\right\} .
\end{aligned}
$$

If $\operatorname{dim} S=\operatorname{dim} \mathbf{h}$, then the Lie algebra of the isometry group is $\mathbf{g}$ (the Lie algebra of $G$ ).

Proof. Use the notation in section 3. If $E=\rho_{*}(\mathbf{e})$ where $\mathbf{e} \in \mathbf{h}$, then $E \in S$, so $\operatorname{dim} S \geq \operatorname{dim} \mathbf{h}$. Suppose that $\tilde{G}$ is the isometry group of $(G / H, \eta)$. To prove the lemma, it is sufficient to show $\operatorname{dim} \tilde{G}=\operatorname{dim} G$. Let $\tilde{H} \subset \tilde{G}$ be the isotropy subgroup at $\sigma=[H] \in G / H$ with linear isotropy representation $\tilde{\rho}$. We have $G \subset \tilde{G}$, $\operatorname{dim} G=n+\operatorname{dim} H$, and $\operatorname{dim} \tilde{G}=n+\operatorname{dim} \tilde{H}$. By the argument just given, $\tilde{\rho}_{*}(\tilde{\mathbf{h}})$ satisfies (6.2). Therefore, if the hypothesis of the theorem hold then $\operatorname{dim} \tilde{\mathbf{h}}=\operatorname{dim} \mathbf{h}$ and $\operatorname{dim} G=\operatorname{dim} \tilde{G}$.

The set $S$ is the Lie algebra of the stabilizer of both the curvature tensor and its covariant derivative at a point. This lemma states that if this subalgebra has the same dimension as $\mathbf{h}$, then the isometry algebra can not have dimension greater than $n+\operatorname{dim} \mathbf{h}$. Therefore, it must be the given algebra. Lemma 6.3 of course generalizes using the higher order covariant derivatives of the curvature tensor.

We can now prove the converse condition in Theorem 2.4 by using Lemmas 6.2 , Lemma 6.3, and the computations from section 5 . That is, for each Lie algebra pair in the theorem we find values for the coefficients of the curvature form $\Omega$ such that Lemma 6.3 is satisfied.

Proof. Proof of converse for Theorem 2.4: We start with the Lorentz signature.

Case 3: If $C_{13}^{1}\left(p_{1}{ }^{2}+4 p_{2}{ }^{2}\right)^{-1}=L \neq 0$, then Lemma 6.3 is satisfied, and the isometry algebra is $\mathbf{A} 4$.

Subcase 4.1: If $t_{2}-2 t_{1}{ }^{2}=L \neq 0$ then Lemma 6.3 is satisfied, and the algebra is A1.

Subcase 4.2.a: If $p_{7}+t^{2} p_{4} \neq 0$ then Lemma 6.3 is satisfied, and the algebra is $\mathbf{A 2}$.

Subcase 4.2.b: If $L=C_{13}^{1}+p_{7} p_{4} \neq 0$ then Lemma 6.3 is satisfied, and the algebra is $\mathbf{A} 3$.

Now we consider the signature $(2,2)$ cases.

Subcase 2.1: If $t \neq 0$ then Lemma 6.3 is satisfied, and the isometry algebra is B2.

Subcase 2.2: See the first part of the of Theorem 2.5 in section 7 .

Case 3: If $r t-s^{2} \neq 0$ then Lemma 6.3 is satisfied, and the isometry algebra is B1.

Subcase 4.1: If $2 p_{6} t^{2}+p_{1}+p_{7}=K \neq 0$, Lemma 6.3 is satisfied and the isometry algebra is $\mathbf{A 1}$. Subcase 4.2: If $p_{2} \neq 0$ and $2 p_{6} t^{2}+p_{1} \neq 0$, Lemma 6.3 is satisfied and the isometry algebra is $\mathbf{A 2}$. Subcase 4.3: If $C_{13}^{1}-p_{1} p_{6}+p_{6}^{2} \neq 0$, Lemma 6.3 is satisfied, and the isometry algebra is $\mathbf{A 3}$.

\section{The Einstein Examples}

In this section, we prove Theorem 2.5 by constructing all homogeneous Einstein and Ricci flat metrics on the simply connected non-reductive homogeneous spaces of dimension four.

Proof. (Theorem 2.5) By Lemma 5.1, the Lie algebra pair $(\mathbf{g}, \mathbf{h})$ of $G / H$ is isomorphic to $\mathbf{B 3}$ if $\eta$ is Ricci-flat, otherwise it is isomorphic to the Lie algebra pair $\mathbf{A 2}$ with $\alpha=\frac{2}{3}$. To prove the theorem, it is sufficient to construct the two simply connected homogeneous spaces that have Lie algebra pair B3 or A3 with $\alpha=\frac{2}{3}$, and find all the invariant metrics. Theorem 2.4 says that the manifolds themselves are diffeomorphic to $\mathbb{R}^{4}$.

We remind the reader that the Lie algebra of infinitesimal generators of $G$ acting on $G / H$ is isomorphic to the Lie algebra of right invariant vector fields, and so we use a basis of left invariant 
forms $\sigma$ which have structure constants negative to the ones in Theorem 2.2 or 2.3 to construct our examples.

We start by proving the second part of the theorem using the Lie algebra in A2. The (negative of the) structure equations are easily integrated on $\mathbb{R}^{5}$ to give the left-invariant forms

$\sigma^{5}=d x^{5}, \sigma^{4}=e^{-x^{5}} d x^{4}, \sigma^{3}=e^{(1-\alpha) x^{5}} d x^{3}, \sigma^{2}=e^{-\alpha x^{5}}\left(d x^{2}+y^{3} d x^{4}\right), \sigma^{1}=e^{-(\alpha+1) x^{5}}\left(d x^{1}-x^{4} d x^{2}\right)$.

Let $X_{i}, i=1 \ldots 5$ be the dual frame of left invariant vector fields. The $X_{4}$ basic symmetric bilinear forms on $G$ are easily computed to be

$$
\tilde{\eta}=a_{1}\left(2 \sigma^{1} \sigma^{3}-\left(\sigma^{2}\right)^{2}\right)+a_{2}\left(\sigma^{3}\right)^{2}+2 a_{3} \sigma^{3} \sigma^{5}+a_{4}\left(\sigma^{5}\right)^{2} .
$$

With the coordinates $y^{1}=x^{1}+x^{3}\left(x^{4}\right)^{2} / 2, y^{2}=x^{2}+x^{3} x^{4}, y^{3}=x^{3}$, and $y^{4}=x^{5}$ on the quotient of $G$ by the orbits of $X_{4}=e^{x^{5}}\left(\partial_{x^{4}}-x^{4} x^{3} \partial_{x^{1}}-x^{3} \partial_{x^{2}}\right)$ we have $\tilde{\eta}=\pi^{*} \eta$ where

$$
\eta=a_{1} e^{-2 \alpha y^{4}}\left(2 d y^{1} d y^{3}-\left(d y^{2}\right)^{2}\right)+a_{2} e^{2(1-\alpha) y^{4}}\left(d y^{3}\right)^{2}+2 a_{3} e^{(1-\alpha) y^{4}} d y^{3} d y^{4}+a_{4}\left(d y^{4}\right)^{2} .
$$

These bi-linear forms are non-degenerate if $a_{1} a_{4} \neq 0$. If $a_{2} \neq 0$ the only Killing vectors are

$Y_{1}=\partial_{y^{1}}, Y_{2}=\partial_{y^{2}}, Y_{3}=\partial_{y^{3}}, Y_{4}=y^{2} \partial_{y^{1}}+y^{3} \partial_{y^{2}}, Y_{5}=(1+\alpha) y^{1} \partial_{y^{1}}+\alpha y^{2} \partial_{y^{2}}+(\alpha-1) y^{3} \partial_{y^{3}}+\partial_{y^{4}}$

These vector fields form a Lie algebra with the multiplication table in A2. At the point $(0,0,0,0)$ the isotropy is $Y_{4}$, and $\eta$ is the most general metric invariant under the flow of these Killing fields. When $\alpha=\frac{2}{3}$, these metrics are Einstein and they are not of constant curvature when $a_{2} \neq 0$. The signature of theses metrics can be only Lorentz or $(2,2)$, as expected.

For the Lie algebra pair in B3, consider the quotient space $G / H$ where $G=S L(2, \mathbb{R}) \ltimes \mathbb{R}^{2} \oplus \mathbb{R}$ and

$$
\left.H=\left\{\left(\begin{array}{ll}
1 & 0 \\
t & 1
\end{array}\right),\left(\begin{array}{l}
0 \\
s
\end{array}\right), s\right) \quad t, s \in \mathbb{R}\right\} .
$$

The manifold $G / H$ is diffeomorphic to $\mathbb{R}^{2}-(0,0) \times \mathbb{R}^{2}$. In terms of coordinates $\left(x^{1}, x^{2}, x^{3}, x^{4}\right)$ on $G / H$ the most general metric having signature $(2,2)$ on $G / H$ where $G$ acts by isometries is

$$
\hat{\eta}=2 \sigma^{1} \sigma^{2}+2 \sigma^{3} \sigma^{4}
$$

where $\sigma^{1}=d x^{1}, \sigma^{2}=d x^{4}+\frac{L x^{2}}{2}\left(x^{2} d x^{1}-x^{1} d x^{2}\right), \sigma^{3}=d x^{2}, \sigma^{4}=-d x^{3}+\frac{L x^{1}}{2}\left(x^{1} d x^{2}-x^{2} d x^{1}\right)$. Let $\pi: \mathbb{R}^{4} \rightarrow M$ be given by $x^{1}=e^{y^{1}} \cos y^{2}, x^{2}=e^{y^{2}} \sin y^{2}, x^{3}=y^{3}, x^{4}=y^{4}$, and $\eta=\pi^{*} \hat{\eta}$. This gives the metrics $\eta$ in part one of Theorem 2.5. The covering group $\tilde{G}=\tilde{S L}(2, \mathbb{R}) \ltimes \mathbb{R}^{2} \oplus \mathbb{R}$ acts transitively and by isometries on $\mathbb{R}^{4}$ for the metrics $\eta$. There are eight Killing vector fields for $\eta$ :

$$
\begin{aligned}
& Y_{1}=\cos \left(2 y^{2}\right) \partial_{y^{1}}-\sin \left(2 y^{2}\right) \partial_{y^{2}}+y^{3} \partial_{y^{3}}-y^{4} \partial_{y^{4}}, Y_{2}=\frac{1}{2} \sin \left(2 y^{2}\right) \partial_{y^{1}}+\cos ^{2} y^{2} \partial_{y^{2}}+y^{3} \partial_{y^{4}} \\
& Y_{3}=\frac{1}{2} \sin \left(2 y^{2}\right) \partial_{y^{1}}-\sin ^{2}\left(y^{2}\right) \partial_{y^{2}}+y^{4} \partial_{y^{3}}, Y_{4}=\partial_{y^{4}}, Y_{5}=-\partial_{y^{3}}, Y_{6}=e^{y^{1}} \cos \left(y^{2}\right) \partial_{y^{3}}+e^{y^{1}} \sin y^{2} \partial_{y^{4}}, \\
& Y_{7}=e^{-y^{1}} \cos \left(y^{2}\right) \partial_{s^{1}}-e^{y^{1}} \sin \left(y^{2}\right) \partial_{s^{2}}+L e^{2 y^{1}}\left(\frac{1}{2} \sin \left(2 y^{2}\right) \partial_{y^{3}}+\sin ^{2}\left(y^{2}\right) \partial_{y^{4}}\right) \\
& Y_{8}=e^{-y^{1}} \sin \left(y^{2}\right) \partial_{s^{1}}+e^{y^{1}} \cos y^{2} \partial_{s^{2}}-L e^{2 y^{1}}\left(\cos ^{2}\left(y^{2}\right) \partial_{y^{3}}+\frac{1}{2} \sin \left(2 y^{2}\right) \partial_{y^{4}}\right) .
\end{aligned}
$$

The first six of these vector fields are complete and are the infinitesimal generators for the transitive action of $\tilde{G}$ on $\mathbb{R}^{4}$. The metric $\eta$ is the most general metric invariant under the flow of these six 
Killing fields. In order to have a complete Killing vector field of the form $a X_{7}+b X_{8}$, it is necessary and sufficient that the differential equations

$$
\frac{d y^{1}}{d t}=e^{-y^{1}}\left(a \cos y^{2}+b \sin y^{2}\right), \frac{d y^{2}}{d t}=e^{-y^{1}}\left(b \cos y^{2}-a \sin y^{2}\right)
$$

admit solutions defined for all $t \in \mathbb{R}$. By letting $z=y^{1}-i y^{2}$, this equation becomes

$$
\frac{d z}{d t}=(a+i b) e^{-z}
$$

which has solution $z=\ln \left((a+i b) t+\left(c_{0}+i c_{1}\right)\right)$. For any initial condition of the form $z(0)=r(a+i b)$ where $r \in \mathbb{R}$, the solution does not exist for all $t \in \mathbb{R}$. Therefore, the Lie algebra of the isometry group is $\mathbf{s l}(2, \mathbb{R}) \ltimes \mathbb{R}^{2} \oplus \mathbb{R}$, and at the point $(0,0,0,0)$ the isotropy subalgebra is $\left\{Y_{3}, Y_{5}+Y_{6}\right\}$. This proves the first part of the theorem. For completeness, the non-zero components of the curvature form are

$$
\Omega_{3}^{1}=-\Omega_{2}^{3}=-3 L \hat{\sigma}^{1} \wedge \hat{\sigma}^{3},
$$

where $\hat{\sigma}^{i}=\pi^{*} \sigma^{i}$. By using this formula for the curvature form, the Ricci tensor is found to vanish. It is worth noting, that the covariant derivative of the Riemann curvature vanishes.

\section{Concluding Remarks}

In this paper we have investigated only the most basic questions surrounding the geometry of non-reductive homogeneous spaces. We have not addressed such natural problems as determining the holonomy of these spaces [6], the homogeneous structure of the geodesics of theses spaces [12], or whether they are geodesically complete. These problems will be considered in future research.

\section{Appendix A. Some Computations in a BASIS}

We write out the conditions in Lemma 3.2 in a basis. Let $\left\{\mathbf{b}_{\alpha}\right\}_{\alpha=1 \ldots q}$ be a basis for the subalgebra $\mathbf{h} \subset \mathbf{o}(p, \tilde{p})$ and complete this to a basis $\left\{\tilde{\mathbf{b}}_{r}, \mathbf{b}_{\alpha}\right\}_{r=1 \ldots n(n-1) / 2-q, \alpha=1 \ldots q}$ for $\mathbf{o}(p, \tilde{p})$. In this basis, the structure constants are

$$
\left[\mathbf{b}_{\alpha}, \mathbf{b}_{\beta}\right]=K_{\alpha \beta}^{\gamma} \mathbf{b}_{\gamma}, \quad\left[\mathbf{b}_{\alpha}, \mathbf{b}_{r}\right]=\tilde{K}_{\alpha r}^{\beta} \mathbf{b}_{\beta}+\hat{K}_{\alpha r}^{s} \mathbf{b}_{s}, \quad\left[\mathbf{b}_{r}, \mathbf{b}_{s}\right]=\tilde{L}_{r s}^{\alpha} \mathbf{b}_{\alpha}+L_{r s}^{t} \mathbf{b}_{t} .
$$

Let $\left\{\mathbf{e}_{\alpha}\right\}_{\alpha=1 \ldots q}$, form a basis for $\mathbf{h} \subset \mathbf{g}=\mathbf{h} \oplus \mathbb{R}^{n}$, where $\rho_{*}\left(\mathbf{e}_{\alpha}\right)=\mathbf{b}_{\alpha}$, and complete this to a basis $\left\{\tilde{\mathbf{e}}_{i}, \mathbf{e}_{\alpha}\right\}_{1 \leq i \leq n, 1 \leq \alpha \leq q}$ for $\mathbf{g}$. Let $\left\{\theta^{i}, \omega^{\alpha}\right\}_{i=1 \ldots n, \alpha=1 \ldots q}$ be the dual basis. We may then write

$$
\omega=\omega^{\alpha} \mathbf{b}_{\alpha}+\tilde{\omega}^{r} \tilde{\mathbf{b}}_{r}, \quad \Omega=\Omega^{\alpha} \mathbf{b}_{\alpha}+\tilde{\Omega}^{r} \tilde{\mathbf{b}}_{r}
$$

where $\omega^{\alpha}, \tilde{\omega}^{r}, \Omega^{\alpha}$, and $\tilde{\Omega}^{r} \in \mathbf{g}^{*}$. By conditions $\left.\hat{2}\right]$ and $\left.\hat{3}\right]$, these forms satisfy

$$
\omega^{\alpha}\left(\mathbf{e}_{\beta}\right)=\delta_{\beta}^{\alpha}, \quad \tilde{\omega}^{r}\left(\mathbf{e}_{\alpha}\right)=0, \quad \Omega^{\alpha}\left(\mathbf{e}_{\alpha}\right)=0, \quad \tilde{\Omega}^{r}\left(\mathbf{e}_{\alpha}\right)=0 .
$$

Consequently, we may write

$$
\tilde{\omega}^{r}=P_{i}^{r} \theta^{i}, \quad \Omega^{\alpha}=\Omega_{i j}^{\alpha} \theta^{i} \wedge \theta^{j}, \quad \tilde{\Omega}^{r}=\tilde{\Omega}_{i j}^{r} \theta^{i} \wedge \theta^{j}
$$

where $P_{i}^{r}, \Omega_{i j}^{\alpha}$, and $\tilde{\Omega}_{i j}^{r} \in \mathbb{R}$. By using $\left.\hat{2}\right]$ and $\left.\hat{3}\right]$, we also have

$$
\iota_{\mathbf{e}_{\alpha}} d \theta^{i}=-b_{\beta j}^{i} \omega^{\beta}\left(\mathbf{e}_{\alpha}\right) \wedge \theta^{j}=-b_{\alpha j}^{i} \theta^{j}
$$


where $\mathbf{b}_{\alpha}=\left(b_{\alpha j}^{i}\right)$. From this equation, it follows

$$
d \theta^{i}=-b_{\alpha j}^{i} \omega^{\alpha} \wedge \theta^{j}-b_{r[k}^{i} P_{j]}^{r} \theta^{j} \wedge \theta^{k} .
$$

By (A.1), $\omega=\omega^{\alpha} \mathbf{b}_{\alpha}+P_{i}^{r} \theta^{i} \mathbf{b}_{r}$. Substituting this into $\iota_{\mathbf{e}_{\alpha}} \Omega=0$ in $\left.\hat{4}\right]$ the coefficients of $\mathbf{b}_{r}$ give

$$
P_{i}^{r} b_{\alpha j}^{i}-\hat{K}_{\alpha s}^{r} P_{j}^{s}=0
$$

The coefficients of $\mathbf{b}_{\beta}$ in $\iota_{\mathbf{e}_{\alpha}} \Omega=0$ give

$$
\iota_{\mathbf{e}_{\alpha}} d \omega^{\beta}+K_{\beta \gamma}^{\alpha} \omega^{\gamma}+\tilde{K}_{\alpha r}^{\beta} P_{i}^{r} \theta^{i}=0 .
$$

This equation leads to the formula

$$
d \omega^{\alpha}=-\frac{1}{2} K_{\beta \gamma}^{\alpha} \omega^{\beta} \wedge \omega^{\gamma}-\tilde{K}_{\beta r}^{\alpha} P_{i}^{r} \omega^{\beta} \wedge \theta^{i}-\frac{1}{2} C_{j k}^{\alpha} \theta^{j} \wedge \theta^{k}
$$

where $C_{j k}^{\alpha}=C_{[j k]}^{\alpha}$ are yet to be determined. The form $\Omega$ can be computed from (A.4) and (A.3):

$$
\Omega=\left(P_{i}^{r} P_{k}^{s} \tilde{b}_{s j}^{i}+\frac{1}{2} L_{s t}^{r} P_{j}^{s} P_{k}^{t}\right) \theta^{j} \wedge \theta^{k} \otimes \mathbf{b}_{r}+\frac{1}{2}\left(\tilde{L}_{r s}^{\alpha} P_{i}^{r} P_{j}^{s}-C_{i j}^{\alpha}\right) \theta^{i} \wedge \theta^{j} \otimes \mathbf{b}_{\alpha}
$$

By choosing $P_{i}^{r}$ and $C_{j k}^{i}$, we can satisfy the last two equations in $\left.\hat{4}\right]$ (the Bianchi identities). The Bianchi identities can be imposed by either computing $\Omega$ and implementing them as written in $\hat{4}]$ or imposing $d^{2} \theta^{i}=0$ and $d^{2} \omega^{\alpha}=0$ in (A.2) and (A.4).

We write, in terms of our basis, the condition for the algebra $\mathbf{g}$ to be reductive.

Lemma A1. Let $G \rightarrow G / H$ with $H$ be a homogeneous space with $H$ connected and where the Lie algebra $\mathbf{g}$ admits forms $\theta$ and $\omega$ satisfying the conditions in Lemma 3.2. Then $G \rightarrow G / H$ is reductive if and only if there exists constants $r_{i}^{\alpha}$ such that

$$
r_{i}^{\gamma} K_{\beta \gamma}^{\alpha}-r_{i}^{\alpha} b_{\beta j}^{i}=\tilde{K}_{\beta r}^{\alpha} P_{i}^{r}
$$

Proof. Since $H$ is connected, the form $\left(\omega^{\beta}+r_{i}^{\beta} \theta^{i}\right) \otimes \mathbf{e}_{\beta}$ is invariant (or equivariant) if and only if its Lie derivative with respect to $\mathbf{e}_{\alpha} \in \mathbf{h}$ is zero. Therefore, $G / H$ is reductive if and only if there exists $r_{i}^{\beta}$ such that

$$
\iota_{\mathbf{e}_{\alpha}}\left(d \omega^{\beta}+r_{i}^{\beta} d \theta^{i}\right) \otimes \mathbf{e}_{\beta}+\left(\omega^{\beta}+r_{i}^{\beta} \theta^{i}\right) \otimes K_{\alpha \beta}^{\gamma} \mathbf{e}_{\gamma}=0
$$

Expanding this equation out using (A.2), (A.3), and (A.4) we get equation (A.5).

\section{THANKS.}

The authors would like to thank Ian Anderson for numerous helpful suggestions and for the use of his package Vessiot available at www.math.usu.edu/ $f g \_m p$. We also thank Charlie Torre, Stephen Yeung, and Scot Adams for helpful discussions. 


\section{REFERENCES}

1. L. Bérard-Bergery, Les espaces homogènes Riemanniens de dimension 4, Textes Mathématiques 3 (1981), 40-60.

2. A. Besse, Einstein Manifolds, Springer-Verlag, 1987.

3. É. Cartan, Leçons sur la géométrie des espaces de Riemann, Gauthier-Villars, 1951.

4. C. Chevalley, On the Topological Structure of Solvable Groups, Ann. of Math. 42(3) (1941), 668-675.

5. P.M. Gadea, J.A. Oubina, Reductive homogeneous pseudo-Riemannian manifolds, Monatsh. Math. 124(1) (1997), 17-34.

6. R. Ghanam,G. Thompson, The holonomy Lie algebras of neutral metrics in dimension four, J. Math. Phys. 42(5) (2001), 2266-2284.

7. S. Ishihara, Homogeneous Riemannian Spaces of Four Dimension, J. Math. Soc. Japan 7 (1955), 345-370.

8. G. Jensen, Geometry of Homogeneous Einstein Spaces of Dimension Four, Jour. Diff. Geom. 3 (1969), 309-349.

9. S.S. Kobyashi, K. Nomizu, Foundations of Differential Geometry Vol. I, John Wiley, 1996.

10. B. Komrakov Jr., Einstein-Maxwell equation on four-dimensional homogeneous spaces, Lobachevskii Journal of Mathematics 8 (2001), 33-165.

11. T.H. Koornwinder, Invariant differential operators on nonreductive homogeneous spaces. Afdeling Zuivere Wiskunde [Department of Pure Mathematics] 153 (1981), 1-15; http://arXiv.org/abs/math/0008116.

12. O. Kowalski, J. Szenthe, On the existence of homogeneous geodesics in homogeneous Riemannian manifolds, Geometriae Dedicata 1-2 (2000), 209-214.

13. G.D. Mostow, Factor Spaces of Solvable Groups, Ann. of Math. 60 (1) (1954), 1-27.

14. A.L. Onishchik (Ed.), Lie Groups and Lie Algebras, Springer-Verlag, 1998.

15. J. Patera, P. Winternitz, H. Zassenhaus, Continuous subgroups of the fundamental groups of physics I. General method and the Poincaré group., J. Math. Phys. 16(8) (1975), 1597-1614.

16. J. Patera, R.T. Sharp, P. Winternitz, H. Zassenhaus, Continuous subgroups of the fundamental groups of physics III. The de-Sitter groups, J. Math. Phys. 18(12) (1977), 2259-2288.

17. J. Patera, R.T. Sharp, P. Winternitz, H. Zassenhaus, Invariants of real low dimension Lie algebras, J. Math. Phys. 17(6) (1976), 986-994.

18. A.Z. Petrov, Einstein Spaces, Pergamon Press, 1969.

19. J. Wolf, Spaces of Constant Curvature, Publish or Perish, 1984.

Department of Mathematics and Statistics, Utah State University, Logan Utah, 84322

E-mail address: fels@math.usu.edu,arenner@cc.usu.edu 